The Interpersonal Effects of Emotions in Money versus Candy Games

Xijing Wang and Eva G. Krumhuber

University College London

Jonathan Gratch

University of Southern California

\begin{abstract}
Author Note
Correspondence concerning this article should be addressed to Xijing Wang, Department of Experimental Psychology, University College London, 26 Bedford Way, London WC1H 0AP, United Kingdom. E-mail: xijing.wang.13@ucl.ac.uk
\end{abstract}

Word Count: 10990 


\begin{abstract}
Emotional expressions significantly influence perceivers' behavior in economic games and negotiations. The current research examined the interpersonal effects of emotions when such information cannot be used to guide behavior for increasing personal gain and when monetary rewards are made salient. For this, a one-shot Public Goods Game (Studies 1, 2, and 3) and Dictator Game (Studies 4 and 5) were employed, in which the dominant strategy to maximize personal payoff is independent from the counterplayers' intention signaled through their facial expressions (happiness, sadness, and anger). To elicit a monetary mindset, we used money (vs. candy) as the mode of exchange in the games with (Studies 1 and 2) or without (Studies 3, 4, and 5) additional contextual framing (i.e. Wall Street Game vs. Community Game). Across five studies $(N=1211)$, participants were found to be more generous towards happy and sad targets compared to angry ones. Such behavioral response based on emotional information was accounted for by the trait impressions (i.e. likability, trustworthiness) formed of the counterplayer. This effect was significantly reduced when money acted as the mode of exchange, thereby making participants focus more on their selfgain. Together, the findings extend previous work by highlighting the social functional role of emotions in human exchange and its moderation by money as a transaction medium.
\end{abstract}

Keywords: emotion; facial expression; decision-making; person perception; money; marketpricing 


\section{The Interpersonal Effects of Emotions in Money versus Candy Games}

Our decisions are not only guided by personal feelings and emotions, but also by those of other people with whom we interact (Van Kleef, 2009). In recent years, the interpersonal effects of emotion on social behavior (Van Kleef, De Dreu \& Manstead, 2010) have become increasingly acknowledged. Empirical evidence has demonstrated that behavior in negotiation settings and bargaining (e.g. Van Dijk, Van Kleef, Steinel, \& Van Beest, 2008; Van Kleef, De Dreu, \& Manstead, 2004), as well as in economic games (e.g. de Melo, Carnevale, Read, \& Gratch, 2014; Krumhuber et al., 2007; Van der Schalk, Kuppens, Bruder, \& Manstead, 2015) can be shaped by the emotional displays of another person (i.e. counterpart).

However, the overwhelming majority of these studies were structured in a way such that participants and their interaction partner(s) are interdependent by having the ability to mutually influence each other's material outcome. In those situations, the emotions expressed by the counterplayers are also often directed at participants or their behavior during interaction (e.g. Adam \& Brett, 2015; Côté, Hideg, \& Van Kleef, 2013; Lelieveld, Van Dijk, Van Beest, Steinel, \& Van Kleef, 2011; Sinaceur \& Tiedens, 2006). Thus, the emotional displays offer crucial information with respect to participants' private payoff chances. Attending to these signals and adjusting one's behavior accordingly facilitates personal gain. The question then arises whether people are still sensitive to their interaction partners' emotions when those cannot be used to guide behavior for maximizing self-gain. And does such emotional responsivity vary with the type of exchange between two partners?

Universally, money acts as the medium of exchange. The prevalence of money as an incentive can be observed not only in laboratory settings but also in real life. While people generally assume that money serves as the canonical object of desire for motivating human decision-making (Lea \& Webley, 2006), the presence of money naturally activates a market- 
pricing mode (Fiske, 1992; Mead \& Stuppy, 2014). As a result, it facilitates self-serving but impairs other-oriented behaviors (e.g. Kasser, 2016; Zaleskiewicz, Gasiorowska, \& Vohs, 2017). The second aim of this research, therefore, was to study whether money as a transaction medium moderates perceivers' responses to an interaction partner's expressed emotion.

\section{Social Functions of Emotion and Trait Perception}

People not only have subjective emotional experiences, but also express emotions to communicate how they feel and think (Van Kleef, 2009). These emotional signals can be observed by one's interaction partner and have the potential to crucially impact their behavior (Van Kleef, 2009). The idea that emotions act as an interpersonal source of information is captured by the interpersonal approach to emotions, which is also in line with the social functions perspective of emotions. Accordingly, people are social by nature and express emotions as a useful means to coordinate social decision-making situations (Keltner \& Haidt, 1999). The emotional displays in turn help observers to understand others' social intentions (e.g. affiliate, dominate, or signal the need for help), thereby guiding behavior towards efficient social coordination.

In this vein, a growing body of research on the interpersonal effects of emotions has demonstrated that behavioral responses can be significantly shaped by another person's emotion. This was typically shown to be the case when two or more interaction partners are interdependent, i.e. they rely on each other to achieve mutual gain, and/or the emotion expressed by one party is directed at another person or his/her behavior (e.g. Adam \& Brett, 2015; Côté et al., 2013; Lelieveld et al., 2011; Pietroni, Van Kleef, De Dreu, \& Pagliaro, 2008; Steinel, Van Kleef, \& Harinck, 2008; Van Dijk et al., 2008). In those situations, 
understanding a partner's emotion and adjusting one's behavior accordingly allows one to achieve optimal coordination, which in turn facilitates personal gain.

While facial expressions can be used to predict a target's immediate behavior during interaction, such displays may also be seen as informative with respect to long-term behavioral tendencies as in the case of trait perceptions (Knutson, 1996). In this context, inferences from emotional expressions are overgeneralized to judgments of the person's character. This could apply especially to incidental emotions which are not directed at any particular person or event, thereby potentially signaling to others long-term dispositional traits (Knutson, 1996). Consistent with this notion, it has been shown that happiness and sadness elicit higher ratings of trustworthiness than does anger, probably because they communicate the general intention for affiliation and social closeness (e.g. Hess, Blairy, \& Kleck, 2000; Sutherland, Young, \& Rhodes, 2017). As such, it seems possible that facial emotions impact others' judgement and behavior in the absence of any interdependence between two parties and when information about the interaction partner is limited such as in zero acquaintance situations (Kenny, 1994). Indeed, there is evidence showing that people are more likely to offer help to strangers who appear likable and attractive (e.g. West \& Brown, 1975; Wilson, 1978) and punish more severely those who look untrustworthy (e.g. Porter, ten Brinke, \& Gustaw, 2010; Wilson \& Rule, 2015). For the present research, we therefore tested whether participants would base their decisions on trait information (e.g.., trustworthiness, likability) derived from the counterplayer's facial expression, even when there is no need to rely on it for optimizing personal gain.

\section{The Moderating Role of Money}

People establish different forms of relationships to meet their varied needs (Fiske, 1992). Market pricing mode is one of the fundamental modes people use to construct their 
social relations, thereby shaping how they perceive and interact with others (Fiske, 1992). Given that transactions outside one's close social circle, i.e. via economic trade, are beneficial for securing goods and services, market pricing has emerged with the advent of money. As a result, relationships in which money acts as the medium of exchange or salient cue are guided by measurable metrics which allow for direct cost-benefit calculations (Mead \& Stuppy, 2014).

In line with this notion, empirical evidence has demonstrated that money affects people's attitudes and responses to emotions. Activating the concept of money, for example by exposing people to monetary cues (i.e. words and images), decreases the tendency to express emotions and leads to more unfavorable reactions to others' emotions (Jiang, Chen, \& Wyer, 2014). Further research showed that money-related incentives or thoughts reduce the accuracy of emotional inferences (Ma-Kellams, \& Blascovich, 2013), compassion towards unfortunate others (Molinsky, Grant, \& Margolis, 2012; Stellar, Manzo, Kraus, \& Keltner, 2012), and perspective-taking (Sheldon \& Kasser, 1995; Van Laer, De Ruyter, \& Cox, 2013). They also undermine motives to perceive a mind in irrelevant targets (Wang \& Krumhuber, 2017) and impair theory of mind ability (Ridinger \& McBride, 2015).

These detrimental effects could largely be due to the fact that money makes people prioritize themselves, while in parallel it reduces their intention for interpersonal closeness and bonding. For example, it was found that individuals who are chronically in a monetary mindset (e.g. economics students and people who highly value money) or those situated in a monetary relation (i.e. consumers) tend to behave more selfishly and feel less responsible for their selfish acts (Bauer, Wilkie, Kim, \& Bodenhausen, 2012; Sheldon \& McGregor, 2000; Wang, Malhotra, \& Murnighan, 2011). Furthermore, basketball and hockey players were shown to engage in more self-serving behaviors during their final year of contract when money is naturally more salient relative to previous years (Beus \& Whitman, 2017). Rather 
than nourishing intimacy (e.g. Kasser \& Ryan, 2001; Kushlev, Dunn, \& Ashton-James, 2012), people seem to construe personal relations with others in an instrumental manner (Andrighetto, Baldissarri, \& Volpato, 2017; Teng, Chen, Poon, Zhang, \& Jiang, 2016). In consequence, communal and other-oriented behaviors like caring and helpfulness are impaired by money (DeVoe \& Pfeffer, 2007; Gasiorowska, Zaleskiewicz, \& Wygrab, 2012; Roberts \& Roberts, 2012). If this assumption holds for the present research, the tendency to consider affective responses is likely to be reduced when money is made salient. As a result, people in the monetary mode should be less affected by their partner's emotion when making decisions.

\section{The Present Research}

While there is converging evidence for the impact of emotional displays on decisionmaking when two parties are mutually dependent, the present research first aimed to examine the interpersonal effects of emotions when a) those are incidental, i.e. they occur in the absence of an emotion-eliciting event; hence, they mainly serve as trait information, and b) the counterparts' emotions cannot guide behavior for maximizing personal payoff, i.e. there is no real interdependency between the players. The second aim of this research was to study whether money as a transaction medium moderates such effects.

To this end, we employed two commonly used economic games: the Public Goods Game (PGG) and Dictator Game (DG). While the dominant strategy to maximize personal payoff is independent from others' intention in the one-shot version of the PGG, participants' reliance on the counterplayer is further reduced in the DG. The counterparts' expressions in the games systematically differed in terms of their emotions and portrayed either happiness, sadness or anger. While happiness conveys an intention for affiliation and cooperation, sadness signals the need for help and elicits compassion, both indicating a potential for social 
closeness. By contrast, anger communicates threat and hostility (e.g. Hess et al., 2000; McLellan, Johnston, Dalrymple-Alford, \& Porter, 2010; Krumhuber \& Manstead, 2009). Despite the fact that the emotion portrayals are not relevant for guiding behaviors to maximize personal payoff, we expected them to exert basic social functions in the formation of trait impressions (e.g. Fischer \& Manstead, 2008; Knutson, 1996), resulting in higher offers being made in response to happy and sad counterparts compared to angry ones.

To elicit a monetary mindset, we used money as the mode of exchange in the game with (Studies 1 and 2) or without (Studies 3, 4, and 5) additional contextual framing (i.e. Wall Street Game). Situational labels such as the name of the game can shape people's behaviors by evoking different norms of behavior (Ellingsen, Johannesson, Mollerstrom, \& Munkhammar, 2012; Liberman, Samuels, \& Ross, 2004). Interestingly, these behavioral patterns are activated not only by explicit descriptions of the task - so-called social framing effects - but also by subtler cues indicative of the type of relational mode (e.g. Kay, Wheeler, Bargh, \& Ross, 2004). Given its utility and role as a medium for economic transaction, we therefore expected money or monetary incentives to activate a market-pricing mindset (e.g. DeVoe \& Iyengar, 2010; Heyman \& Ariely, 2004; Jin \& Huang, 2014). This was contrasted with a control condition that involved the exchange of another resource (i.e. candy) with equivalent monetary value in the game (for a similar procedure see Gasiorowska, Chaplin, Zaleskiewicz, Wygrab, \& Vohs, 2016; Heyman \& Ariely, 2004; Liberman et al., 2004). If money encourages people to focus on self-gain (e.g. Mead \& Stuppy, 2014; Zaleskiewicz et al., 2017), contribution rates should be lower in the money than candy condition. This should be the case in particular when facing happy and sad targets, given the reduced intention for social closeness. Importantly, we predicted participants' behavior in the money condition to be less affected by the counterparts' emotions given that such emotional signals are not directly informative for maximizing personal payoff. 
In Studies 1 and 2, participants played a Public Goods Game (i.e. PGG, one-shot version) with counterplayers who expressed either happiness, sadness, or anger on their profile images. The game was described either as Wall Street Game or Community Game (control condition) and involved money or candy as the medium of exchange. Study 3 aimed to replicate the findings and further test whether money (vs. candy) as an experimental currency in itself, without the contextual framing of the game, is sufficient to drive differences in responses. Study 4 was designed to extend the findings of the previous experiments with a different economic game, i.e. Dictator Game (DG, one-shot version), in which the seeming interdependence between the players is further reduced. Finally, the aim of Study 5 was to replicate the findings using a between-subjects design and examine whether the effects generalize to counterplayers of both genders. In addition, we examined two potential mechanisms that could account for the effects of emotion (happiness, sadness, anger) and condition (money, candy) on allocation decisions: the trait impressions formed of the counterplayer (likability, trustworthiness) and participants' attentiveness to themselves (i.e. self-gain focus).

Sample size calculation. Given the aim of the current study is to test the main effect of emotion (happiness, sadness, and anger) on decisions and whether such effect further varies as a function of condition (money, candy), we expected a main effect of emotion (Study 1-4, within-subjects; Study 5, between-subjects) as well as an interaction between emotion and condition (between-subjects). Sensitivity power analyses revealed that minimum effect sizes of $f=.11($ Study $1, N=145), f=.13($ Study $2, N=108), f=.12($ Study $3, N=$ $121), f=.13($ Study $4, N=118), f=.12$ (Study $5, N=718)$ could be detected under standard criteria (i.e., $\alpha=.05$ two-tailed, $\beta=.80, r=0.5$ (correlation among measures), non-sphericity correction $=0.8$, respectively. 


\section{Study 1}

Study 1 examined whether people are influenced by the counterplayers' expressed emotions even when those signals cannot guide their behavior to maximize personal gain. In addition, we tested whether such effects are altered in situations that imply a monetary exchange. To this end, we employed a two-person Public Goods Game (PGG) and manipulated the type of exchange, i.e. money (experimental condition) vs. candy (control condition), together with the name of the game, i.e. Wall Street vs. Community, respectively. The game is structured in a way such that participants have to choose how many of their private tokens to put into a public pool. The tokens in the pool are multiplied by a certain factor and then evenly divided among the players. Each player also keeps the tokens s/he does not contribute (Gunnthorsdottir, Houser, \& McCabe, 2007). In the current study, participants played multiple one-shot versions of this game, each time with a different counterpart whose facial expressions conveyed either happiness, sadness, or anger.

If participants were purely rational agents with the aim to maximize their payoff, the dominant strategy would be to contribute zero regardless of whatever the other person does. In addition, their contribution level should not be affected by the counterpart's emotion. Based on existing evidence, however, this hardly occurs, showing that people don't act in a completely utilitarian manner (e.g. Camerer, 2003). In line with the social functions perspective of emotions, we expected participants' contribution rates to be significantly higher when facing happy and sad counterparts compared to angry ones. Moreover, this effect should be shaped by the game context, resulting in lower offer rates and responses that are driven less by others' facial expressions when money is salient.

\section{Method}


Participants and Design. A total of 145 participants (57 women, $M_{\text {age }}=33.9, S D=$ 9.31, 86\% Caucasian, 14\% others) were recruited via Amazon's Mechanical Turk. The twofactor experimental design included the game condition (Wall Street/money vs. Community/candy) as a between-subjects variable, and the counterpart's emotion (anger, happiness, sadness) as a within-subjects variable. Participants were randomly assigned to one of the two conditions, resulting in seventy-two people in the money condition and seventythree people in the candy condition. Participants received $\$ 2$ as compensation at the end of the study. The study was conducted with ethical approval from the Department of Experimental Psychology at University College London, United Kingdom. Across all five studies, we report all measures, manipulations, and exclusions.

Materials. Frontal facial shots depicting happiness, sadness, and anger were selected from previously validated databases (Langner et al., 2010; Olszanowski et al., 2015; Van der Schalk, Hawk, Fischer, \& Doosje, 2011) and served as the counterparts' profile images. Each emotion was portrayed by five different Caucasian women, representing fifteen counterparts with unique facial identity. Pilot-testing with a separate group of participants $(N=32)$ showed high recognition rates for the target emotions (happiness: 96\%, anger: $98 \%$, and sadness: $87 \%$ ) in a forced-choice task with the six basic emotions, relief, envy, and 'other emotion' as answer options. For the present study, we also included portrayals of five women with a neutral expression to act as filler stimuli.

Procedure and Measures. The game task was run using Qualtrics, a web-based software (Provo, UT). After reporting basic demographic data, participants were informed that they were going to play a two-person Wall Street Game (money condition)/ Community Game (candy condition) multiple times, each time with a different player. We told them that all players had pre-registered at our game website, with their facial behavior and response patterns being systematically recorded. Importantly, it was made clear that this was not a live 
game. Hence, the interaction between participants and their counterplayers was not in realtime.

Participants learned that players would be endowed at the beginning of each game round with 100 points of private money/candy. Players could then decide how many of their private money/candy points to contribute to a shared account. The points being contributed would be multiplied by 1.2 and evenly divided between the two players. Players were able to keep the points which they did not share. It was made clear that independent from the behavior of the other player, the more they wanted themselves to earn, the less they should put into the public pot. In contrast, the more they wanted the other player to earn, the more they should publicly contribute. To increase the level of engagement, we emphasized that the money/candy points they earned in the game would determine their chance to win an additional money bonus (experimental condition)/ candy box (control condition) at the end of the experiment. The value of both rewards was well-matched, i.e. the money bonus and candy box equaled approximately $\$ 20$. Although we did not mention the price of the candy box so as to avoid a mixed market mode (see Heyman \& Ariely, 2004), participants were presented with an image of it (50 flavor gift box $600 \mathrm{~g}$ by Jelly Belly®) which indirectly conveyed the respective value.

After some comprehension checks ${ }^{1}$, participants repeatedly played the PGG (i.e. 20 one-shot trials). In each round, a money/candy image (1280 x 720 pixels) appeared in the center of the screen to signal the start of a game trial. This was followed by a waiting page which ranged in duration from 8 to $15 \mathrm{~s}$. Next, a facial image ( $400 \times 300$ pixels) of their ostensible counterpart appeared and remained visible until a 'Next' button was pressed that led to the decision-making page. Participants made their offers by deciding on the number of money/candy points to contribute to the public pot, ranging from 0 to 100 with an increment of 10 points. For subsequent analyses, the contribution rates were re-labeled using a scale 
Running head: MONEY AND EMOTIONS

from $0 \%$ (lowest) to $100 \%$ (highest). No immediate feedback was provided in terms of how many points they gained each time. Instead, we told participants that they would be informed about their final payoffs at the end of the game. The order of presentation of the counterparts' images was randomized across the 20 game trials. In the end, all participants were debriefed and received a fixed amount of $\$ 2$.

\section{Results and Discussion}

Contribution rates for each type of emotion were averaged across the five face exemplars and submitted to a 3 (emotion: anger, happiness, sadness) x 2 (condition: Wall Street/money, Community/candy) analysis of variance (ANOVA), with the latter variable being a between-subjects factor. The Greenhouse-Geisser adjustment to the degrees of freedom was applied when the assumption of sphericity was violated.

As predicted, there was a significant main effect of emotion, $F(1.53,219)=47, p$ $<.001, \eta_{\mathrm{p}}{ }^{2}=.246$, such that participants' contribution levels were highest in response to happy targets $(M=30.4 \%, S D=35.6), t(144)=4.38, p<.001, d=0.37$ (happiness vs. sadness); $t(144)=7.52, p<.001, d=0.63$ (happiness vs. anger), followed by sad targets $(M=$ $24.8 \%, S D=32.5), t(144)=6.42, p<.001, d=0.54$ (sadness vs. anger), and lastly angry targets $(M=14.6 \%, S D=27.3)$. There was also a trend toward lower contribution rates in the money $(M=19.1 \%, S D=28.6)$ compared to the candy condition $(M=27.3 \%, S D=30.4)$, $F(1,143)=2.80, p=.097, \eta_{\mathrm{p}}^{2}=.019^{2}$.

In addition, a significant interaction between emotion and condition emerged, $F(1.53$, $219)=7.58, p=.002, \eta_{\mathrm{p}}^{2}=.050$. The contribution pattern of those in the money condition was less likely to be affected by the counterparts' emotions than those in the candy condition (see Figure 1). Although allocations in response to the three emotions significantly differed from each other in both conditions, such differences were smaller in the money than candy 
condition. This applied in particular to the comparison between happiness and anger (money: $M_{\text {difference }}=9.6 \%, t(71)=4.04, p<.001, d=0.48 ;$ candy: $M_{\text {difference }}=22 \%, t(72)=6.61, p$ $<.001, d=0.78)$ as well as sadness and anger (money: $M_{\text {difference }}=5.4 \%, t(71)=2.99, p$ $=.004, d=0.35$; candy: $\left.M_{\text {difference }}=14.9 \%, t(72)=5.98, p<.001, d=0.70\right)$, but not necessarily to the comparison between happiness and sadness (money: $M_{\text {difference }}=4.2 \%, t(71)$ $=3.61, p=.001, d=0.43$; candy: $\left.M_{\text {difference }}=7.0 \%, t(72)=3.08, p=.003, d=0.36\right)$. Pairwise comparisons also revealed that participants allocated fewer points to happy $\left(M_{\text {money }}=23.7 \%\right.$, $S D_{\text {money }}=32.5$ vs. $\left.M_{\text {candy }}=37.0 \%, S D_{\text {candy }}=37.5, t(143)=-2.27, p=.025, d=-0.38\right)$ and sad targets $\left(M_{\text {money }}=19.5 \%, S D_{\text {money }}=30.0\right.$ vs. $M_{\text {candy }}=29.9 \%, S D_{\text {candy }}=34.1, t(143)=-1.96, p$ $=.052, d=-0.32$ ) when they were in the money than candy condition, whereas the difference was not significant in response to angry targets $\left(M_{\text {money }}=14.1 \%, S D_{\text {money }}=27.3\right.$ vs. $M_{\text {candy }}=$ $\left.15.0 \%, S D_{\text {candy }}=27.4, t(143)=-0.21, p=.838, d=-0.03\right)$.

Although the counterplayers' emotions were not directly relevant for guiding behaviors to maximize personal gain, participants were significantly affected by this information. Moreover, such effect was less pronounced for people in the money compared to the candy condition. While allocations varied as a function of the three emotions in both conditions, such differences were smaller when the game was about money. In addition, participants in the money (vs. candy) condition, were less generous when facing targets showing happiness and sadness, i.e. expressions that signal a potential for social closeness.

\section{Study 2}

Study 2 aimed to replicate the findings of Study 1 in the laboratory and with a different sample, i.e. college undergraduate students.

\section{Method}


Participants and Design. A total of 108 undergraduate students from University of Southern California (72 women, $M_{\text {age }}=20.3, S D=2.69$, 34.8\% Caucasian, $31.3 \%$ Asian, 13.3\% Latino, 20\% others) were recruited through the Sona Systems participant panel. The two-factor experimental design included the game condition (Wall Street/money vs. Community/candy) as a between-subjects variable, and the counterpart's emotion (anger, happiness, sadness) as a within-subjects variable. Participants were randomly assigned to one of the two conditions, resulting in fifty-three people in the money condition and fifty-five people in the candy condition. Participants either received $\$ 5$ (money condition) or 5 bags of candies (equivalent to $\$ 5$, candy condition) as compensation at the end of the study. The study was conducted with ethical approval from the University of Southern California, United States.

Materials. These were identical to Study 1.

Procedure and Measures. These were the same as in Study 1 except that participants were paid in terms of the game currency (i.e. cash or candy), supposedly determined by the money/candy points they had earned during the game. In order to increase the salience of the experimental currency, money notes/candies were placed on a small table next to participants' workstation. Although the candy price was not explicitly mentioned so as to avoid a mixed market mode, the value of money (in the form of $\$ 1$ notes) and candies (in the form of \$1 per jelly bean bag) was well matched.

\section{Results and Discussion}

Contribution rates for each type of emotion were averaged across the five corresponding exemplars and submitted to a 3 (emotion: happiness, sad, anger) x 2 (condition: money, candy) ANOVA, with the latter variable being a between-subjects factor. 
Running head: MONEY AND EMOTIONS

The Greenhouse-Geisser adjustment to the degrees of freedom was applied when the assumption of sphericity was violated.

Replicating the findings of Study 1, there was a significant main effect of emotion, $F(1.90,202)=38.7, p<.001, \eta_{\mathrm{p}}^{2}=.267$, such that people's contribution levels were generally higher in response to happy $(M=34.2 \%, S D=23.9)$ and $\operatorname{sad}(M=31.8 \%, S D=$ 23.5) compared to angry targets $(M=21.2 \%, S D=21.0), t(107)=7.44, p<.001, d=0.72$ (happiness vs. anger); $t(107)=6.79, p<.001, d=0.66$ (sadness vs. anger), whereas the difference between happy and sad targets was marginal significant, $t(107)=1.71, p=.091, d$ $=0.17$. In addition, participants in the money condition $(M=24.8 \%, S D=19.8)$ made on average lower contributions than those in the candy condition $(M=33.1 \%, S D=21.0), F(1$, 106) $=4.46, p=.037, \eta_{\mathrm{p}}^{2}=.040$.

Replicating the findings of the Study 1, a significant interaction between emotion and condition emerged, $F(1.90,202)=3.71, p=.028, \eta_{\mathrm{p}}{ }^{2}=.034$, showing that contribution rates were less likely to be affected by the counterparts' emotions in the money than candy condition (see Figure 2). Although allocations in response to the three emotions were often significantly different from each other in both conditions, such differences were smaller in the money than candy condition. This applied in particular to the comparison between happiness and anger (money: $M_{\text {difference }}=9.6 \%, t(52)=4.99, p<.001, d=0.67$; candy: $\left.M_{\text {difference }}=16.4 \%, t(54)=5.75, p<.001, d=0.77\right)$ as well as sadness and anger $($ money: $M_{\text {difference }}=6.6 \%, t(52)=3.39, p=.001, d=0.46$; candy: $M_{\text {difference }}=14.5 \%, t(54)=6.22, p$ $<.001, d=0.84)$, but not necessarily to the comparison between happiness and sadness $\left(\right.$ money: $M_{\text {difference }}=3.0 \%, t(52)=2.07, p=.043, d=0.28 ;$ candy: $M_{\text {difference }}=1.9 \%, t(54)=$ $0.78, p=.437, d=0.11)$. Further pairwise comparisons revealed that people's contribution level was lower in response to happy $\left(M_{\text {money }}=29.0 \%, S D_{\text {money }}=22.5, M_{\text {candy }}=39.2 \%\right.$, $\left.S D_{\text {candy }}=24.2, t(106)=-2.27, p=.025, d=-0.44\right)$ and sad targets $\left(M_{\text {money }}=26.0 \%, S D_{\text {money }}\right.$ 
$\left.=21.8, M_{\text {candy }}=37.3 \%, S D_{\text {candy }}=24.0, t(106)=-2.55, p=.012, d=-0.49\right)$ when they were in the money than candy condition, whereas the group difference was not significant in response to angry ones $\left(M_{\text {money }}=19.4 \%, S D_{\text {money }}=19.0, M_{\text {candy }}=22.8 \%, S D_{\text {candy }}=22.8, t(106)=-\right.$ $0.84, p=.402, d=-0.16)$.

Together, the findings of Studies 1 and 2 provide consistent evidence for the impact of emotional expressions on participants' decisions in the PGG. In general, contribution rates were significantly higher in response to happy and sad targets compared to angry ones. Moreover, responses varied with the type of game condition. When money (compared to candy) was made salient through contextual framing and mode of exchange, participants were less likely to take the emotional information of the counterpart's face into account, resulting in lower levels of emotion-based responses.

\section{Study 3}

The purpose of Study 3 was to replicate the findings of Studies 1 and 2 and further test whether the experimental currency in itself, without any contextual framing of the game, is sufficient to trigger distinct behavioral responses. To this end, we employed the same PGG game as in Studies 1 and 2 and used money or candy (control condition) as the transaction medium. It was hypothesized that contribution rates would be significantly higher towards happy and sad counterparts than angry ones. Such responses which derive from others' facial expression should further be reduced when money, as compared to candy, acts as the mode of exchange in the game.

\section{Method}

Participants and Design. 121 participants (54 women, $M_{\text {age }}=33.7, S D=9.96,80.8 \%$ Caucasian, 5.5\% Latin American, $14.7 \%$ other) were recruited via Amazon's Mechanical 
Turk. The two-factor experimental design included the experimental currency (money vs. candy) as a between-subjects variable, and the counterpart's emotion (anger, happiness, sadness) as a within-subjects variable. Participants were randomly assigned to one of the two conditions. This resulted in approximately sixty people in each condition. All participants received a fixed payment of $\$ 2$ at the end of the study. The study was conducted with ethical approval from the Department of Experimental Psychology at University College London, United Kingdom.

Materials. These were identical to Studies 1 and 2.

Procedure and Measures. These were the same as in Studies 1 and 2 except that the scenario was described as a money (money condition) or candy game (control condition). To rule out the possibility that the present effects are solely driven by the desirability of the incentive type, participants' incentive motivation was assessed prior to the start of the experiment on a 1 (not at all) to 7 (very much) scale, by asking how motivated they would be by money/candy in the game.

\section{Results and Discussion}

Contribution rates for each type of emotion were averaged across the five face exemplars and submitted to a 3 (emotion: happiness/sad/anger) x 2 (condition: money, candy) ANOVA. The Greenhouse-Geisser adjustment to the degrees of freedom was applied when the assumption of sphericity was violated.

Replicating the findings of the previous two studies, the results revealed a significant main effect of emotion, $F(1.91,226)=27.4, p<.001, \eta_{\mathrm{p}}{ }^{2}=.187$. In general, contribution rates were highest in response to happy targets $(M=35.7 \%, S D=34.0), t(120)=4.14, p$ $<.001, d=0.41$ (happiness vs. sadness); $t(120)=6.84, p<.001, d=0.61$ (happiness vs. anger), followed by sad targets $(M=28.2 \%, S D=31.9), t(120)=3.28, p=.001, d=0.22$ 
Running head: MONEY AND EMOTIONS

(sadness vs. anger), and lastly angry targets $(M=22.9 \%, S D=29.7)$. Also, participants' contributions were lower in the money $(M=23.3 \%, S D=28.7)$ compared to the candy condition $(M=34.5 \%, S D=30.2), F(1,119)=4.41, p=.038, \eta_{\mathrm{p}}^{2}=.036$.

Replicating the findings of Studies 1 and 2, a significant interaction between emotion and condition emerged, $F(1.91,226)=5.87, p=.004, \eta_{\mathrm{p}}{ }^{2}=.047$. This interaction remained significant when incentive motivation was entered as a covariate in the analysis, $F(1.91,225)$ $=4.63, p=.012, \eta_{\mathrm{p}}{ }^{2}=.038$, showing that contribution patterns of those in the money condition were less likely to be affected by counterparts' emotions than those in the candy condition(see Figure 3). Although allocations in response to the three emotions were often significantly different from each other in both conditions, such differences were smaller in the money than candy condition. This applied in particular to the comparison between happiness and anger (money: $M_{\text {difference }}=9.0 \%, t(59)=4.08, p<.001, d=0.53$; candy: $\left.M_{\text {difference }}=16.6 \%, t(60)=5.61, p<.001, d=0.72\right)$ as well as sadness and anger (money: $M_{\text {difference }}=-0.6 \%, t(59)=-0.36, p=.721, d=-0.05 ;$ candy: $M_{\text {difference }}=11.1 \%, t(60)=4.35$, $p<.001, d=0.56$ ), but not necessarily to the comparison between happiness and sadness $\left(\right.$ money: $M_{\text {difference }}=9.6 \%, t(59)=2.97, p=.004, d=0.38 ;$ candy: $M_{\text {difference }}=5.5 \%, t(60)=$ $3.24, p=.002, d=0.42$ ). Pairwise comparisons also revealed that participants in the money condition were less generous towards happy $\left(M_{\text {money }}=29.5 \%, S D_{\text {money }}=34.3, M_{\text {candy }}=\right.$ $\left.41.9 \%, S D_{\text {candy }}=32.9, t(119)=-2.04, p=.044, d=-0.37\right)$ and sad targets $\left(M_{\text {money }}=19.9 \%\right.$, $\left.S D_{\text {money }}=29.4, M_{\text {candy }}=36.4 \%, S D_{\text {candy }}=32.4, t(119)=-2.94, p=.004, d=-0.53\right)$ compared to those in the candy condition, whereas this difference was not significant for angry targets $\left(M_{\text {money }}=20.5 \%, S D_{\text {money }}=28.2, M_{\text {candy }}=25.3 \%, S D_{\text {candy }}=31.2, t(119)=-0.89, p=.375, d=\right.$ $-0.16)$.

In line with the findings from Studies 1 and 2, people's decisions were shaped by the counterplayers' expressed emotions even when such information could not be used for 
guiding behavior to maximize personal payoff. Moreover, money made participants less reliant on the emotional information of counterparts' faces, resulting in reduced levels of emotion-based responding in resource allocation. These effects occurred when experimental currency was varied devoid of any manipulation of the game label, suggesting that money as a medium of exchange per se can shape behavioral responses.

\section{Study 4}

Study 4 aimed to extend the existing findings with a different type of economic game in which the seeming relevance of counterplayers' emotional displays for maximizing personal payoff was further removed. For this, a one-shot Dictator Game (DG) was employed. The game requires participants to act in the role of an allocator who determines how to split an endowment between themselves and another person (i.e. counterpart). The counterpart's role as the recipient is entirely passive, by simply accepting the allocations that are made by the allocator. If people were purely rational and self-interested agents, they should offer nothing to the other person, and their allocation patterns shouldn't vary as a function of the counterpart's facial emotion. Similar as in Study 3, the game only differed in terms of its experimental currency (money vs. candy).

It was hypothesized that allocation rates would be higher towards happy and sad counterparts than angry ones. However, such responses guided by counterparts' emotional expressions should be significantly reduced when money acts as the medium of exchange. In addition, we obtained ratings of counterparts' likability so as to provide initial evidence for the impact of emotional displays on generic impressions of the other player and how this relates to people's allocation patterns.

\section{Method}


Participants and Design. 118 participants (34 women, $M_{\mathrm{age}}=29.8, S D=9.85,78.8 \%$ Caucasian, 5.1\% Hispanic, 7.6\% Asian, and 8.5\% others) were recruited via Amazon's Mechanical Turk. The two-factor experimental design included the type of experimental currency (money vs. candy) as a between-subjects variable, and the counterpart's emotion (anger, happiness, sadness) as a within-subjects variable. Participants were randomly assigned to one of the two conditions, resulting in approximately sixty people in each condition. All participants received a fixed payment of $\$ 2$ at the end of the study. The study was conducted with ethical approval from the Department of Experimental Psychology at University College London, United Kingdom.

Procedure. The game task was run using Qualtrics, a web-based software (Provo, UT). After reporting basic demographic data, participants were informed that they were going to play a two-person game multiple times, each time with a different player. Similar to previous studies, we told participants that all players had pre-registered at our game website, with their facial behavior being systematically recorded.

Participants learned that allocators in the game were endowed with 100 points of cash/candy which they could unilaterally split between another player (recipient) and themselves. Recipients had to accept any offer that was made. The decision who acts as the allocator and recipient in the game was supposedly determined by chance. To increase participants' motivation towards the incentive, it was emphasized that the money/candy points they earned in the game would determine the chance of getting an extra money bonus/ candy gift voucher at the end of the experiment. As such, the more they gained in the game, the more likely they were to win the prize. In line with the previous studies, the value of the money bonus and candy box were well-matched (both equaled approximately \$20), although the price of the candy box was not explicitly mentioned. 
After some comprehension checks ${ }^{3}$, participants repeatedly played the DG (i.e. 20 one-shot trials) in the role of the allocator. For each trial, a money/candy image (1280 x 720 pixels) appeared in the center of the screen to signal the start of a trial. This was followed by a facial image ( $400 \times 300$ pixels) of their ostensible counterpart. The stimulus material was identical to that used in the previous studies.

Measures. For each counterpart, participants first judged the perceived likability of the person on a 7- point scale ranging from 1 (very dislikable) to 7 (very likable). Next, they made their DG offer by deciding on a split of the 100 money/candy points between themselves and the counterpart, ranging from 100 for self ( 0 for the other) to 0 for self (100 for the other) with an increment of 10 points. For subsequent analyses, the allocation scores were re-labeled using a scale from $0 \%$ (lowest) to $100 \%$ (highest). The order of presentation of the counterparts' images was randomized across the 20 game trials.

After the game, participants' incentive motivation was assessed on a 1 (not at all) to 7 (very much) scale, by asking how motivated they were by money/candy during the game. Finally, participants were debriefed, paid, and thanked.

\section{Results and Discussion}

Allocation scores and likability ratings were averaged for each type of emotion across the five face exemplars and submitted to a 3 (emotion: anger, happiness, sadness) x 2 (condition: money, candy) ANOVA, with the latter variable being a between-subjects factor. The Greenhouse-Geisser adjustment to the degrees of freedom was applied when the assumption of sphericity was violated.

Allocation Scores. Replicating the findings of the previous three studies, there was a significant main effect of emotion, $F(1.69,196)=31.0, p<.001, \eta_{\mathrm{p}}{ }^{2}=.211$, such that allocation rates were highest in response to happy targets $(M=19.4 \%, S D=21.9), t(117)=$ 
4.16, $p<.001, d=0.46$ (happiness vs. sadness), $t(117)=6.31, p<.001, d=0.57$ (happiness vs. anger), followed by sad targets $(M=10.6 \%, S D=16.7), t(117)=4.40, p<.001, d=0.41$ (sadness vs. anger), and lastly angry targets $(M=10.6 \%, S D=16.7)$. Also, participants in the money condition $(M=10.5 \%, S D=15.9)$ made on average less generous offers than those in the candy condition $(M=19.9 \%, S D=19.5), F(1,116)=8.17, p=.005, \eta_{\mathrm{p}}^{2}=.066$.

These two main effects were qualified by a significant interaction between emotion and condition, $F(1.69,196)=8.48, p<.001, \eta_{\mathrm{p}}^{2}=.068$. The interaction remained significant when incentive motivation was entered as a covariate in the analysis, $F(1.69,194)=7.33, p$ $=.002, \eta_{\mathrm{p}}{ }^{2}=.060$. Consistent with the previous findings, allocation patterns were less likely to be affected by counterparts' emotions when money was salient (see Figure 4). Although allocations in response to the three emotions were often significantly different from each other in both conditions, such differences were smaller in the money than candy condition. This applied in particular to the comparison between happiness and anger (money: $M_{\text {difference }}$ $=4.6 \%, t(59)=2.86, p=.006, d=0.37 ;$ candy: $M_{\text {difference }}=12.9 \%, t(57)=6.09, p<.001, d=$ 0.79 ) as well as sadness and anger (money: $M_{\text {difference }}=1.1 \%, t(59)=1.16, p=.250, d=0.15$; candy: $\left.M_{\text {difference }}=8.7 \%, t(57)=4.59, p<.001, d=0.59\right)$, but not necessarily to the comparison between happiness and sadness (money: $M_{\text {difference }}=3.6 \%, t(59)=3.07, p=.003$, $d=0.40$; candy: $\left.M_{\text {difference }}=4.3 \%, t(57)=2.86, p=.006, d=0.37\right)$. In addition, participants in the money condition offered fewer points to happy $\left(M_{\text {money }}=13.3 \%, S D_{\text {money }}=19.1, M_{\text {candy }}\right.$ $\left.=25.6 \%, S D_{\text {candy }}=23.0, t(116)=-3.18, p=.002, d=-0.58\right)$ and sad targets $\left(M_{\text {money }}=9.7 \%\right.$, $\left.S D_{\text {money }}=15.9, M_{\text {candy }}=21.3 \%, S D_{\text {candy }}=22.1, t(116)=-3.29, p=.001, d=-0.60\right)$ than those in the candy condition, whereas this difference was not significant for angry targets $\left(M_{\text {money }}=8.6 \%, S D_{\text {money }}=15.4, M_{\text {candy }}=12.7 \%, S D_{\text {candy }}=18.0, t(116)=-1.32, p=.190, d=\right.$ $-0.24)$. 
Likability Ratings. An ANOVA showed that there was a significant main effect of emotion, $F(1.66,193)=182.8, p<.001, \eta_{\mathrm{p}}^{2}=.612$, such that happy targets were judged as most likable $(M=5.03, S D=1.18), t(117)=12.3, p<.001, d=1.12$ (happiness vs. sadness), $t(117)=15.5, p<.001, d=1.35$ (happiness vs. anger), followed by sad targets $(M=3.55, S D$ $=1.10), t(117)=8.91, p<.001, d=0.66$ (sadness vs. anger), and lastly angry targets $(M=$ $2.57, S D=1.36)$. The main effect of condition and the interaction between emotion and condition were not significant, $F(1,116)=0.35, p=.553, \eta_{\mathrm{p}}{ }^{2}=.003 ; F(2,115)=2.64, p$ $=.076, \eta_{\mathrm{p}}^{2}=.044$

Importantly, a mixed-effects model, with allocation scores as the dependent variable, participants as a random factor and likability ratings as a fixed factor, revealed that ratings of likability were significantly related to levels of generosity in the game, $B=0.37, S E=0.03$, $95 \%$ CI $[0.31,0.44], t(249)=12.0, p<.001, d=1.12$.

The present results replicate the findings of Studies 1, 2, and 3 with the use of a different type of economic game, i.e. DG. Even when participants were not dependent on the information provided by their counterplayer's emotion to maximize payoff, they were still significantly influenced by it. When the situation implied a monetary exchange, however, participants were less likely to be affected by the counterparts' emotions in their resource allocations. Similar as in Study 3, these effects occurred when we controlled for incentive motivation and when the game label was absent, suggesting that the type of experimental currency in itself can drive differences in behavioral responding. It was further shown that ratings of likability varied with the counterplayers' displayed emotions and significantly predicted subsequent resource allocations. However, given that facial emotion was treated as a within-subjects factor, we could not directly test for an effect of emotion on allocation decisions via trait impressions of the counterplayer. We aimed to follow up on this point in the final study. 


\section{Study 5}

Study 5 aimed to provide a direct replication of the previous findings using a between-subjects design. Furthermore, to ensure the previous results are not specific to one gender, we extended the stimuli to include both men and women. In order to examine the underlying mechanisms involved in the main and interaction effects of emotion and condition, we considered besides the trait impression formed of the counterplayer (i.e. likability and trustworthiness), participants' focus on self-gain maximization as a potential mediator.

\section{Method}

Participants and Design. 720 participants were recruited via Amazon's Mechanical Turk. Two participants did not finish the study and were thus excluded from the analysis. This left a final sample of 718 participants (353 women, $M_{\text {age }}=35.8, S D=9.57,78.1 \%$ Caucasian). The two-factor experimental design included the type of experimental currency (money vs. candy) and the counterpart's emotion (anger, happiness, sadness) as betweensubjects variables. Participants were randomly assigned to one of the six conditions, resulting in approximately one hundred and twenty people in each condition. All participants were remunerated $(\$ 0.5)$ at the end of the study. The study was conducted with ethical approval from the Department of Experimental Psychology at University College London, United Kingdom.

Materials. The facial images of women depicting happiness, sadness, and anger were identical to those used in previous studies. In addition, frontal facial shots of men depicting happiness, sadness, and anger were selected from the Radboud Faces Database (Langner et al., 2010) and also served as the counterparts' profile images. Each emotion was portrayed by four different Caucasian men and, similar to the exemplars of women, was highly 
Running head: MONEY AND EMOTIONS

recognizable (happiness: $96 \%$, anger: $88 \%$, and sadness: $90 \%$ ) as shown in a pilot study with a separate group of participants $(N=30)$ using a forced-choice task with the six basic emotions, relief, envy, and 'other emotion' as answer options.

Procedure and Measures. The game task was identical to that of Study 4, except that participants in this study played the game only once with a randomly assigned counterplayer whose emotional expression (i.e. happiness, sadness, and anger) systemically differed between participants. Approximately half of the participants played the game with a female counterplayer and the other half played it with a male counterplayer.

Before the start of the game, participants' incentive motivation was assessed on a 1 (not at all) to 7 (very much) scale, by asking how motivated they would be by money/candy in the game. We also asked participants either before or after the game (the order was counterbalanced between participants) to indicate the extent to which they felt driven by a focus on self-gain maximization using a scale from 1 (not at all) to 9 (very much).

Participants made their DG offer by deciding on a split of the 100 money/candy points between themselves and the counterpart, ranging from 100 for self ( 0 for the other) to 0 for self (100 for the other) with an increment of 10 points. For subsequent analyses, these allocation scores were re-labeled using a scale from $0 \%$ (lowest) to $100 \%$ (highest). During the game task, participants also evaluated the perceived likability and trustworthiness of the counterplayer, either before or after the allocation decision (counterbalanced order) using a 7point scale ranging from 1 (very dislikable/ untrustworthy) to 7 (very likable/trustworthy).

In the end, all participants were debriefed, paid, and thanked.

\section{Results and Discussion}

Allocation Scores. Allocation rates were submitted to a 3 (emotion: anger, happiness, sadness) x 2 (condition: money, candy) x 2 (target gender: man, woman) between- 
subjects ANOVA. There was no significant main effect of target gender, $F(1,706)=0.17, p$ $=.678, \eta_{\mathrm{p}}^{2}=.000$, nor any interaction with emotion, $F(2,706)=0.56, p=.572, \eta_{\mathrm{p}}{ }^{2}=.002$, condition, $F(2,706)=0.18, p=.672, \eta_{\mathrm{p}}^{2}=.000$, and emotion by condition, $F(2,706)=0.27$, $p=.766, \eta_{\mathrm{p}}^{2}=.001$. Hence, this factor was excluded from further analysis.

Replicating the findings of the previous four studies, there was a significant main effect of emotion, $F(2,712)=15.7, p<.001, \eta_{\mathrm{p}}{ }^{2}=.042$. As such, allocation rates were highest in response to happy targets $(M=32.6 \%, S D=26.2), t(486)=2.78, p=.006, d=$ 0.25 (happiness vs. sadness), $t(472)=5.74, p<.001, d=0.53$ (happiness vs. anger), followed by sad targets $(M=26.1 \%, S D=25.3), t(472)=2.93, p=.004, d=0.27$ (sadness vs. anger), and lastly angry targets $(M=19.6 \%, S D=23.0)$. Also, participants made on average less generous offers in the money $(M=21.1 \%, S D=23.4)$ than candy condition $(M=30.8 \%, S D$ $=26.3), F(1,712)=26.8, p<.001, \eta_{\mathrm{p}}^{2}=.036$.

There was a significant interaction between emotion and condition, $F(2,712)=6.15$, $p=.002, \eta_{\mathrm{p}}{ }^{2}=.017$, and this effect remained significant when incentive motivation was entered as a covariate in the analysis, $F(2,711)=6.16, p=.002, \eta_{\mathrm{p}}{ }^{2}=.017$. Consistent with the previous findings, allocation patterns were less likely to be affected by counterparts' emotions when money acted as the mode of exchange (see Figure 5). Although allocations in response to the three emotions were often significantly different from each other in both conditions, such differences were smaller in the money than candy condition, This applied in particular to the comparison between happiness and anger (money: $M_{\text {difference }}=6.5 \%, t(220)=$ $1.99, p=.047, d=0.15$; candy: $\left.M_{\text {difference }}=18.5 \%, t(250)=6.04, p<.001, d=0.76\right)$ as well as sadness and anger (money: $M_{\text {difference }}=-1.4 \%, t(222)=-0.46, p=.646, d=-0.02$; candy: $\left.M_{\text {difference }}=13.5 \%, t(248)=4.31, p<.001, d=0.55\right)$, but not necessarily to the comparison between happiness and sadness (money: $M_{\text {difference }}=7.9 \%, t(224)=2.61, p=.010, d=0.22$; candy: $\left.M_{\text {difference }}=5.0 \%, t(260)=1.53, p=.126, d=0.19\right)$. In addition, participants in the 
Running head: MONEY AND EMOTIONS

money condition offered fewer points to happy $\left(M_{\text {money }}=25.9 \%, S D_{\text {money }}=24.6, M_{\text {candy }}=\right.$ $\left.38.3 \%, S D_{\text {candy }}=26.2, t(242)=-3.80, p<.001, d=-0.49\right)$ and sad targets $\left(M_{\text {money }}=18.0 \%\right.$, $\left.S D_{\text {money }}=20.7, M_{\text {candy }}=33.3 \%, S D_{\text {candy }}=26.9, t(242)=-4.93, p<.001, d=-0.64\right)$ than those in the candy condition, whereas this difference was not significant for angry targets $\left(M_{\text {money }}=19.4 \%, S D_{\text {money }}=24.2, M_{\text {candy }}=19.8 \%, S D_{\text {candy }}=22.0, t(228)=-0.15, p=.878, d\right.$ $=-0.02)$.

Trait Impressions. Because likability and trustworthiness ratings were highly correlated, $r(718)=.854, p<.001$, Cronbach's $\alpha=.92$, they were averaged and then submitted to a 3 (emotion: anger, happiness, sadness) x 2 (condition: money, candy) betweensubjects ANOVA ${ }^{4}$. There was a significant main effect of emotion, $F(2,712)=316.4, p$ $<.001, \eta_{\mathrm{p}}{ }^{2}=.471$, such that happy targets evoked the most positive trait impressions $(M=$ $6.77, S D=1.51), t(486)=14.6, p<.001, d=1.32$ (happiness vs. sadness), $t(472)=26.0, p$ $<.001, d=2.38$ (happiness vs. anger), followed by sad targets $(M=4.63, S D=1.72), t(472)$ $=10.4, p<.001, d=0.95$ (sadness vs. anger), and lastly angry targets $(M=3.03, S D=1.63)$. Neither the main effect of condition, $F(1,712)=1.72, p=.190, \eta_{\mathrm{p}}{ }^{2}=.002$, nor the interaction between condition and emotion was significant, $F(2,712)=0.33, p=.719, \eta_{\mathrm{p}}^{2}$ $=.001$.

Focus on Self-Gain. Scores of self-gain focus were submitted to a 3 (emotion: anger, happiness, sadness) x 2 (condition: money, candy) between-subjects ANOVA ${ }^{5}$. A significant main effect of emotion, $F(2,712)=3.62, p=.027, \eta_{\mathrm{p}}{ }^{2}=.010$, revealed that people were less likely to focus on self-gain when facing happy $(M=7.09, S D=2.13)$ compared to angry targets $(M=7.62, S D=1.90), t(472)=-2.86, p=.004, d=-0.26$, with scores for sad targets being in-between $(M=7.32, S D=2.00)$, happiness vs. sadness, $t(486)=1.27, p=.205, d=$ 0.11 ; sadness vs. anger, $t(472)=-1.64, p=.102, d=0.15$. 
Running head: MONEY AND EMOTIONS

Also, focus on self-gain was generally higher in the money $(M=7.84, S D=1.62)$ than candy condition $(M=6.90, S D=2.23), F(1,712)=39.7, p<.001, \eta_{\mathrm{p}}{ }^{2}=.051$. The main effects of emotion and condition were qualified by a significant interaction between the two, $F(2,712)=4.44, p=.012, \eta_{\mathrm{p}}^{2}=.012$. In general, people's attentiveness to self-gain was unaffected by the type of emotional display of their counterplayers when money acted as the mode of exchange, $t \mathrm{~s} \leq 0.50, p \mathrm{~s} \geq .563, d \mathrm{~s} \leq 0.08$. By contrast, participants in the candy condition felt less driven by a focus on self-gain when their counterplayer expressed happiness as opposed to anger, $M_{\text {difference }}=-1.00, t(250)=-3.62, p<.001, d=-0.47$, and sadness compared to anger, $M_{\text {difference }}=-0.66, t(248)=-2.49, p=.014, d=-0.32$; the difference between happiness and sadness was not significant, $t(260)=-1.18, p=.238, d=-$ 0.15 (see Figure 6). In addition, compared to those in the candy condition, people in the money condition were more likely to focus on self-gain when facing happy $\left(M_{\text {money }}=7.81\right.$, $\left.S D_{\text {money }}=1.49, M_{\text {candy }}=6.47, S D_{\text {candy }}=2.39, t(242)=5.16, p<.001, d=0.67\right)$ and sad $\operatorname{targets}\left(M_{\text {money }}=7.91, S D_{\text {money }}=1.51, M_{\text {candy }}=6.81, S D_{\text {candy }}=2.23, t(242)=4.46, p<.001, d\right.$ $=0.58)$, whereas the difference was not significant for angry targets $\left(M_{\text {money }}=7.78, S D_{\text {money }}=\right.$ $\left.1.85, M_{\text {candy }}=7.47, S D_{\text {candy }}=1.93, t(228)=1.26, p=.209, d=0.16\right)$. The result mirrors the pattern for allocation decisions shown above.

Mediation Analysis. To examine whether impression ratings of the counterplayer and participants' self-gain focus mediate the effect of emotion on resource allocation, multiple mediation analyses were conducted. For this, sequential coding was used since participants' offer rates were highest in response to happy $(M=32.6 \%)$, followed by sad $(M=26.1 \%)$, and lastly angry targets $(M=19.6 \%)$. As shown in Figure 7, emotion (D1 and D2) predicted trait impression as well as allocation. Also, trait impression predicted allocation. When controlling for self-gain and impression simultaneously, the effect of emotion on allocation was no longer significant. A bootstrapped analysis (Preacher \& Hayes, 2008; 5,000 resamples) 
revealed that the $95 \%$ confidence interval for the indirect effect did not include zero (D1: CI $[0.34,0.64]$, D2: CI $[0.46,0.83])$. As such, allocation rates were significantly explained by the trait impression derived from counterplayers' expressed emotions. Because emotion did not significantly predict self-gain focus, this variable failed to act as a significant mediator in the model (95\% D1: CI [- 0.05, 0.55], D2: CI [- 0.11, 0.52]).

To examine whether focus on self-gain and impression ratings of the counterplayer further mediate the interaction between emotion and condition on allocation, a mediated moderation model was employed. For this, an interaction term between emotion (sequential coding, D1 and D2) and condition (money = -1, candy =1) was created separately for each contrast code and treated as the independent variable. Trait impression and self-gain were entered simultaneously as potential mediators; allocation was entered as the dependent variable, with emotion (D1 and D2) and condition being considered as covariates (Hayes, 2013). The results showed that focus on self-gain acted as a significant mediator which was able to account for the interaction effect of emotion and condition on resource allocation. As shown in Figure 8, the interaction effect predicted self-gain as well as allocation; self-gain in turn predicted allocation. When controlling for self-gain and impression, the interaction effect of emotion and condition on allocation was no longer significant. A bootstrapped analysis (Preacher \& Hayes, 2008; 5,000 resamples) revealed that the 95\% confidence interval for the indirect effect did not include zero (D1: CI [0.01, 0.21], D2: CI [0.05, 0.26]). Because the interaction term did not significantly predict trait impression, this variable failed to act as a significant mediator in the model (95\% D1: CI [- 0.02, 0.04], D2: CI [- 0.03, 0.03]).

Study 5 extended the previous findings by employing a between-subjects design as well as targets of both genders. In accordance with Studies 1- 4, counterplayers' emotions significantly affected participants in their allocation decisions and formed the basis for trait impressions (perceived likability and trustworthiness) even when those signals were 
Running head: MONEY AND EMOTIONS

irrelevant to payoff maximization. In addition, emotion-based responses were found to be less pronounced in the money than candy condition. This effect was further mediated by participants' focus on their self-gain.

\section{Meta-analysis}

To increase statistical power and improve estimates of the effect size, we performed a within-paper meta-analysis. For this, a random-effects meta-analysis using Comprehensive Meta-Analysis (CMA) software was conducted across the five studies. The results revealed a significant main effect of emotion (happiness, sadness and anger) on allocation rates, $d=$ $0.66,95 \% \mathrm{CI}[0.26,0.36], \mathrm{z}=11.19, p<.001$. Overall, participants were most generous in response to happy targets, $d=0.57,95 \% \mathrm{CI}[0.22,0.33], \mathrm{z}=9.79, p<.001$ (happiness vs. anger); $d=0.28,95 \% \mathrm{CI}[0.09,0.20], \mathrm{z}=4.92, p<.001$ (happiness vs. sadness), followed by sad targets, $d=0.34,95 \% \mathrm{CI}[0.11,0.22], \mathrm{z}=5.82, p<.001$ (sadness vs. anger), and lastly angry targets.

The main effect of condition (money vs. candy) was also significant, $d=-0.38,95 \%$ CI [- 0.27, -0.11$], \mathrm{z}=-4.70, p<.001$. This remained to be the case when allocation rates to neutral targets were included in the analysis, $d=-0.38,95 \% \mathrm{CI}[-0.26,-0.11], \mathrm{z}=-4.76, p$ $<.001$, suggesting that generosity levels generally decreased when money acted as the mode of exchange.

The interaction between emotion and condition was also significant, $d=0.33,95 \% \mathrm{CI}$ $[0.11,0.22], \mathrm{z}=5.75, p<.001$, showing that allocation patterns were less likely to be affected by the emotions of their counterplayers when the game was about money. While participants were more generous in response to sad compared to angry targets in the candy condition, $d=0.60,95 \% \mathrm{CI}[0.21,0.36], \mathrm{z}=7.35, p<.001$, this difference was not significant in the money condition, $d=0.08,95 \% \mathrm{CI}[-0.04,0.12], \mathrm{z}=0.96, p=.339$. When 
Running head: MONEY AND EMOTIONS

comparing allocation rates in response to happy vs. angry targets, the effect was larger in the candy, $d=0.77,95 \%$ CI $[0.29,0.43], \mathrm{z}=9.31, p<.001$, than money condition, $d=0.29$, $95 \%$ CI $[0.06,0.22], \mathrm{z}=3.43, p=.001$. Responses were similar in both conditions and significantly higher for happy compared to sad targets, $d=0.29,95 \%$ CI [0.06, 0.22], z $=$ $3.40, p=.001$ (money); $d=0.24,95 \%$ CI $[0.04,0.19], \mathrm{z}=2.90, p=.004$ (candy). In addition, participants in the money condition were less generous in response to happy, $d=-0.47,95 \%$ CI [- 0.28, - 0.18], $\mathrm{z}=-8.08, p<.001$, and sad targets, $d=-0.56,95 \% \mathrm{CI}[-0.33,-0.22], \mathrm{z}=$ $-9.71, p<.001$, than those in the candy condition, whereas this difference was not significant for angry targets, $d=-0.06,95 \% \mathrm{CI}[-0.09,-0.02], \mathrm{z}=-1.17, p=.244$.

\section{General Discussion}

There is convincing evidence suggesting that people can influence an interaction partner's decision through their facial expressions (e.g., Van Kleef et al., 2004; Wubben, De Cremer, \& Van Dijk, 2009). In the majority of past studies, however, the counterplayers' expressed emotions were critical for determining participants' private payoffs. The current research aimed to extend this work by examining the interpersonal effects of emotions when those cannot be used to increase self-gain, and when money acts a medium of exchange during interaction.

In the context of two economic games, we demonstrated that resource contributions (Studies 1, 2, and 3) and allocations (Studies 4 and 5) were significantly affected by the counterparts' emotions even when those were irrelevant for guiding behaviors for payoff maximization. Consistent with the social functions perspective which suggests that emotions signal different social intentions (e.g. Fischer \& Manstead, 2008), participants made more favorable offers to happy and sad targets compared to angry ones. This was shown to be the case under conditions in which the emotional displays were merely incidental (i.e. unrelated 
to a specific event). Such effect of emotion on decision-making was accounted for by the trait impressions formed of the counterplayer, i.e. happy and sad targets were perceived as more trustworthy and likable than angry ones (Studies 4 and 5). The present finding aligns with evidence from behavioral economics suggesting that humans are not fully rational beings.

Importantly, such behavior changed with the game context. When choosing how much of their private money (as opposed to candy) points to share with other players in a PGG, participants' responses to the emotions of their counterparts significantly decreased when the game was framed as Wall Street Game (vs. Community Game, Studies 1 and 2). This behavioral pattern was replicated with the same game that merely differed in its experimental currency (money vs. candy, Study 3). When a different economic game, i.e. DG, was employed, the mere presence of money was sufficient to reduce the interpersonal effects of counterparts' emotions on allocation decisions (Studies 4 and 5). Such lower emotional responsivity caused by money (i.e. the interaction between emotion and condition on behavior) was found to be mediated by participants' the attentiveness to oneself (i.e. focus on self-gain, Study 5).

Together, the findings provide support for the claim that the influential power of social emotions depends on the situational context of exchange. Specifically, people in monetary markets tend to apply an economic mindset, thereby focusing on themselves and showing reduced other-oriented behavior (e.g. Mead \& Stuppy, 2014; Zaleskiewicz et al., 2017). As a result, money dampens mind perception and perspective-taking (Van Laer et al., 2013; Wang \& Krumhuber, 2017; Wang \& Krumhuber, 2018), making people less empathetic and compassionate (Ma-Kellams \& Blascovich, 2013; Molinsky et al., 2012). This impacts not only people's attitudes towards others' emotion (e.g. Jiang et al., 2014), but also shapes emotion-based decisions. 
Across five studies, there was converging evidence for a reduction in generosity levels when money was involved, which is in accordance with findings reported in the literature (e.g., Marwell \& Ames, 1981; Gasiorowska et al., 2012, 2016; Wang et al., 2011). On average, participants who exchanged money tended to make lower, less generous offers to counterparts. This reduced generosity was particularly evident when facing happy as well as sad counterparts whose expressions signal a potential for social closeness and bonding. Money may therefore induce a mindset that impairs communal orientation and lowers the need for social engagement and bonding (e.g. Burroughs \& Rindfleisch, 2002; Kasser \& Ryan, 2001; Kraus \& Keltner, 2009; Kushlev et al., 2012).

When it comes to practical implications, the present findings suggest that the effects obtained in previous studies may be an underestimation of the social functional power of emotions. While money acts as a salient incentive in negotiation paradigms and economic games, many social relations in daily life are based on non-monetary motives, existing in the absence of clear self-gains. Consequently, one might expect an even stronger interpersonal influence of emotions once people enter a social communal mode of interaction.

Relatedly, our research demonstrates the possibility that using monetary incentives could backfire. Money has been widely adopted to motivate performance both in laboratory settings as well as in real life (e.g., Bonner, Hastie, Sprinkle, \& Young, 2000; Krug, \& Braver, 2014). Based on the present results and those of others (e.g. Gneezy \& Rustichini, 2000; Mellstrom \& Johannesson, 2008; Heyman \& Ariely, 2004), we would predict diverging effects depending on the type and nature of the task. In particular, when altruistic and communal behavior are desirable features, the use of money might lead to diminished or even opposite effects compared to other resources. This insight can be particularly valuable in the context of charitable giving and donation requests. Although happiness and sadness may well be suited as emotional displays to elicit caring and compassion, the salience of money as 
a donation currency, could result in reduced generosity levels compared to other types of inkind goods.

\section{Limitations and Future Outlook}

While the present research examined how the social functions of emotion vary with money as a transaction medium, the counterparts' expressions were designed to be irrelevant to maximizing self-gain. It remains unknown whether these effects would hold or flip once emotions are of instrumental use, e.g. when inferring emotions and the mind of others can increase one's chance to receive monetary rewards. Future studies might examine the moderating role of money (vs. candy) when a) both parties are interdependent by having the ability to mutually influence each other's material payoff and b) counterplayers' emotions are integral to the situation or a direct response to the behavior of participants.

In the present research, candy was chosen as a medium of exchange for the control condition given that it acts as a desirable resource and its value (i.e. price) can be matched with that of money. Although we relied on previous work (Heyman \& Ariely, 2004) in the selection of jelly beans as a form of candy, it must be noted that candy is frequently used as a social gift to signal kindness and the intention for social closeness. The effects obtained in the control condition might therefore be specific to candy. In the future, it will be important to replicate the current findings with a different type of non-monetary incentive that is also less social in its function.

Given that the participants in this research formed a homogenous group of high incentive motivators ( $86.5 \%$ scored $\geq 5$ on a scale from 1 to 7$)$, we did not consider incentive motivation as a potential moderator, but instead controlled for this variable in the statistical analysis (Studies 3, 4, and 5). Nonetheless it is possible that decisions in the economic game are influenced by people's attitudes towards the incentive. For example, existing evidence suggests that the relative desire for money significantly predicts unethical behavior (Tang \& 
Chiu, 2003; Tang \& Chen, 2008) and lower helping behavior (Tang et al., 2008). In future research, it would be interesting to include relevant measures to assess people's chronic desire for incentives as well as other dispositional traits (i.e. Machiavellianism) which might prove relevant in interaction with contextual factors of the game.

\section{Conclusion}

Emotions expressed by others have a powerful impact on the perception and behavior of those who observe them. The present research explored whether people are still sensitive to their counterplayers' emotions when such information cannot be used to maximize selfgain and monetary rewards are made salient. Across five studies, we showed that people's responses are influenced by the emotional displays of their counterparts. However, this effect was reduced in situations that involved the exchange of money. 


\section{References}

Adam, H., \& Brett, J. M. (2015). Context matters: The social effects of anger in cooperative, balanced, and competitive negotiation situations. Journal of Experimental Social Psychology, 61, 44-58.

Andrighetto, L., Baldissarri, C., \& Volpato, C. (2017). (Still) Modern Times: Objectification at work. European Journal of Social Psychology, 47, 25-35.

Bauer, M. A., Wilkie, J. E., Kim, J. K., \& Bodenhausen, G. V. (2012). Cuing consumerism: Situational materialism undermines personal and social well-being. Psychological Science, 23(5), 517-523.

Beus, J. M., \& Whitman, D. S. (2017). Almighty dollar or root of all evil? Testing the effects of money on workplace behavior. Journal of Management, 43(7), 2147-2167.

Bonner, S. E., Hastie, R., Sprinkle, G. B., \& Young, S. M. (2000). A review of the effects of financial incentives on performance in laboratory tasks: Implications for management accounting. Journal of Management Accounting Research, 12(1), 19-64.

Burroughs, J. E., \& Rindfleisch, A. (2002). Materialism and well-being: A conflicting values perspective. Journal of Consumer Research, 29(3), 348-370.

Camerer, C. (2003). Behavioral game theory: Experiments in strategic interaction. Princeton University Press.

Côté, S., Hideg, I., \& van Kleef, G. A. (2013). The consequences of faking anger in negotiations. Journal of Experimental Social Psychology, 49(3), 453-463.

de Melo, C. M., Carnevale, P. J., Read, S. J., \& Gratch, J. (2014). Reading people's minds from emotion expressions in interdependent decision making. Journal of Personality and Social Psychology, 106(1), 73-88.

DeVoe, S. E., \& Iyengar, S. S. (2010). Medium of exchange matters: What's fair for goods is unfair for money. Psychological Science, 21(2), 159-162. 
DeVoe, S. E., \& Pfeffer, J. (2007). Hourly payment and volunteering: The effect of organizational practices on decisions about time use. Academy of Management Journal, 50(4), 783-798.

Ellingsen, T., Johannesson, M., Mollerstrom, J., \& Munkhammar, S. (2012). Social framing effects: Preferences or beliefs? Games and Economic Behavior, 76(1), 117-130.

Faul, F., Erdfelder, E., Lang, A. G., \& Buchner, A. (2007). G* Power 3: A flexible statistical power analysis program for the social, behavioral, and biomedical sciences. Behavior Research Methods, 39(2), 175-191.

Fischer, A. H., \& Manstead, A. S. (2008). The social functions of emotion. In M. Lewis, J. Haviland-Jones, \& L. F. Barrett (Eds.), Handbook of emotions (3rd ed.). New York: Guilford Press.

Fiske, A. P. (1992). The four elementary forms of sociality: framework for a unified theory of social relations. Psychological Review, 99(4), 689-723.

Gasiorowska, A., Chaplin, L. N., Zaleskiewicz, T., Wygrab, S., \& Vohs, K. D. (2016). Money cues increase agency and decrease prosociality among children: Early signs of market-mode behaviors. Psychological Science, 27(3), 331-344.

Gasiorowska, A., Zaleskiewicz, T., \& Wygrab, S. (2012). Would you do something for me? The effects of money activation on social preferences and social behavior in young children. Journal of Economic Psychology, 33(3), 603-608.

Gneezy, U., \& Rustichini, A. (2000). A fine is a price. Journal of Legal Studies, 29, 1-18.

Gunnthorsdottir, A., Houser, D., \& McCabe, K. (2007). Disposition, history and contributions in public goods experiments. Journal of Economic Behavior \& Organization, 62(2), 304-315.

Hayes, Andrew F. (2013). Introduction to Mediation, Moderation, and Conditional Process Analysis: A Regression-Based Approach. New York, NY: The Guilford Press. 
Hess, U., Blairy, S., \& Kleck, R. E. (2000). The influence of facial emotion displays, gender, and ethnicity on judgments of dominance and affiliation. Journal of Nonverbal Behavior, 24(4), 265-283.

Heyman, J., \& Ariely, D. (2004). Effort for payment a tale of two markets. Psychological Science, 15(11), 787-793.

Jiang, Y., Chen, Z., \& Wyer, R. S. (2014). Impact of money on emotional expression. Journal of Experimental Social Psychology, 55, 228-233.

Jin, L., \& Huang, Y. (2014). When giving money does not work: The differential effects of monetary versus in-kind rewards in referral reward programs. International Journal of Research in Marketing, 31(1), 107-116.

Kasser, T. (2016). Materialistic values and goals. Annual Review of Psychology, 67, 489-514.

Kasser, T., \& Ryan, R. M. (2001). Be careful what you wish for: Optimal functioning and the relative attainment of intrinsic and extrinsic goals. In P. Schmuck \& K. M. Sheldon (Eds.), Life goals and well-being: Towards a positive psychology of human striving (pp. 116-131). Goettingen, Germany: Hogrefe \& Huber.

Kay, A. C., Wheeler, S. C., Bargh, J. A., \& Ross, L. (2004). Material priming: The influence of mundane physical objects on situational construal and competitive behavioral choice. Organizational Behavior and Human Decision Processes, 95(1), 83-96.

Keltner, D., \& Haidt, J. (1999). Social functions of emotions at four levels of analysis. Cognition and Emotion, 13(5), 505-521.

Kenny, D. A. (1994). Interpersonal perception: A social relations analysis. Guilford Press.

Knutson, B. (1996). Facial expressions of emotion influence interpersonal trait inferences. Journal of Nonverbal Behavior, 20(3), 165-182.

Kraus, M. W., \& Keltner, D. (2009). Signs of socioeconomic status: A thin-slicing approach. Psychological Science, 20(1), 99-106. 
Krug, M. K., \& Braver, T. S. (2014). Motivation and cognitive control: Going beyond monetary incentives. In The psychological Science of Money (pp. 137-162). Springer New York.

Krumhuber, E. G., \& Manstead, A. S. (2009). Can Duchenne smiles be feigned? New evidence on felt and false smiles. Emotion, 9(6), 807-820.

Krumhuber, E., Manstead, A. S., Cosker, D., Marshall, D., Rosin, P. L., \& Kappas, A. (2007). Facial dynamics as indicators of trustworthiness and cooperative behavior. Emotion, 7(4), 730-735.

Kushlev, K., Dunn, E. W., \& Ashton-James, C. E. (2012). Does affluence impoverish the experience of parenting? Journal of Experimental Social Psychology, 48(6), 13811384.

Langner, O., Dotsch, R., Bijlstra, G., Wigboldus, D. H., Hawk, S. T., \& Van Knippenberg, A. D. (2010). Presentation and validation of the Radboud Faces Database. Cognition and Emotion, 24(8), 1377-1388.

Lea, S. E., \& Webley, P. (2006). Money as tool, money as drug: The biological psychology of a strong incentive. Behavioral and Brain Sciences, 29(2), 161-209.

Lelieveld, G. J., Van Dijk, E., Van Beest, I., Steinel, W., \& Van Kleef, G. A. (2011). Disappointed in you, angry about your offer: Distinct negative emotions induce concessions via different mechanisms. Journal of Experimental Social Psychology, 47(3), 635-641.

Liberman, V., Samuels, S. M., \& Ross, L. (2004). The name of the game: Predictive power of reputations versus situational labels in determining prisoner's dilemma game moves. Personality and Social Psychology Bulletin, 30(9), 1175-1185.

Ma-Kellams, C., \& Blascovich, J. (2013). The ironic effect of financial incentive on empathic accuracy. Journal of Experimental Social Psychology, 49(1), 65-71. 
Marwell, G., \& Ames, R. E. (1981). Economists free ride, does anyone else? Experiments on the provision of public goods, IV. Journal of Public Economics, 15(3), 295-310.

McLellan, T., Johnston, L., Dalrymple-Alford, J., \& Porter, R. (2010). Sensitivity to genuine versus posed emotion specified in facial displays. Cognition and Emotion, 24(8), 12771292.

Mead, N. L., \& Stuppy, A. (2014). Two sides of the same coin: Money can promote and hinder interpersonal processes. In The Psychological Science of Money (pp. 243-262). Springer, New York, NY.

Mellström, C., \& Johannesson, M. (2008). Crowding out in blood donation: was Titmuss right? Journal of the European Economic Association, 6(4), 845-863.

Molinsky, A. L., Grant, A. M., \& Margolis, J. D. (2012). The bedside manner of homo economicus: How and why priming an economic schema reduces compassion. Organizational Behavior and Human Decision Processes, 119(1), 27-37.

Olszanowski, M., Pochwatko, G., Kuklinski, K., Scibor-Rylski, M., Lewinski, P. \& Ohme, R.K. (2015). Warsaw set of emotional facial expression pictures: a validation study of facial display photographs. Frontiers in Psychology, 5, 1516.

Pietroni, D., Van Kleef, G. A., De Dreu, C. K., \& Pagliaro, S. (2008). Emotions as strategic information: Effects of other's emotional expressions on fixed-pie perception, demands, and integrative behavior in negotiation. Journal of Experimental Social Psychology, 44(6), 1444-1454.

Porter, S., ten Brinke, L., \& Gustaw, C. (2010). Dangerous decisions: The impact of first impressions of trustworthiness on the evaluation of legal evidence and defendant culpability. Psychology, Crime, \& Law, 16(6), 477-491. 
Preacher, K. J., \& Hayes, A. F. (2008). Asymptotic and resampling strategies for assessing and comparing indirect effects in multiple mediator models. Behavior Research Methods, 40(3), 879-891.

Ridinger, G., \& McBride, M. (2015). Money affects theory of mind differently by gender. PloS One, 10(12), e0143973.

Roberts, J. A., \& Roberts, C. R. (2012). Money matters: does the symbolic presence of money affect charitable giving and attitudes among adolescents? Young Consumers, 13(4), 329-336.

Sheldon, K. M., \& Kasser, T. (1995). Coherence and congruence: Two aspects of personality integration. Journal of Personality and Social Psychology, 68(3), 531-543.

Sheldon, K. M., \& McGregor, H. A. (2000). Extrinsic value orientation and "the tragedy of the commons". Journal of Personality, 68(2), 383-411.

Sinaceur, M., \& Tiedens, L. Z. (2006). Get mad and get more than even: When and why anger expression is effective in negotiations. Journal of Experimental Social Psychology, 42(3), 314-322.

Steinel, W., Van Kleef, G. A., \& Harinck, F. (2008). Are you talking to me?! Separating the people from the problem when expressing emotions in negotiation. Journal of Experimental Social Psychology, 44(2), 362-369.

Stellar, J. E., Manzo, V. M., Kraus, M. W., \& Keltner, D. (2012). Class and compassion: Socioeconomic factors predict responses to suffering. Emotion, 12(3), 449-459.

Sutherland, C. A., Young, A. W., \& Rhodes, G. (2017). Facial first impressions from another angle: How social judgements are influenced by changeable and invariant facial properties. British Journal of Psychology, 108(2), 397-415. 
Tang, T. L. P., \& Chen, Y. J. (2008). Intelligence vs. wisdom: The love of money, Machiavellianism, and unethical behavior across college major and gender. Journal of Business Ethics, 82(1), 1-26.

Tang, T. L. P., \& Chiu, R. K. (2003). Income, money ethic, pay satisfaction, commitment, and unethical behavior: Is the love of money the root of evil for Hong Kong employees? Journal of Business Ethics, 46(1), 13-30.

Tang, T. L. P., Sutarso, T., Davis, G. M. T. W., Dolinski, D., Ibrahim, A. H. S., \& Wagner, S. L. (2008). To help or not to help? The Good Samaritan Effect and the love of money on helping behavior. Journal of Business Ethics, 82(4), 865-887.

Teng, F., Chen, Z., Poon, K. T., Zhang, D., \& Jiang, Y. (2016). Money and relationships: When and why thinking about money leads people to approach others. Organizational Behavior and Human Decision Processes, 137, 58-70.

Van Der Schalk, J., Hawk, S. T., Fischer, A. H., \& Doosje, B. (2011). Moving faces, looking places: validation of the Amsterdam Dynamic Facial Expression Set (ADFES). Emotion, 11(4), 907-920.

van der Schalk, J., Kuppens, T., Bruder, M., \& Manstead, A. S. (2015). The social power of regret: The effect of social appraisal and anticipated emotions on fair and unfair allocations in resource dilemmas. Journal of Experimental Psychology: General, 144(1), 151-157.

Van Dijk, E., Van Kleef, G. A., Steinel, W., \& Van Beest, I. (2008). A social functional approach to emotions in bargaining: when communicating anger pays and when it backfires. Journal of Personality and Social Psychology, 94(4), 600-614.

Van Kleef, G. A. (2009). How emotions regulate social life the emotions as social information (EASI) model. Current Directions in Psychological Science, 18(3), 184188. 
Van Kleef, G. A., De Dreu, C. K., \& Manstead, A. S. (2004). The interpersonal effects of anger and happiness in negotiations. Journal of Personality and Social Psychology, 86(1), 57-76.

Van Kleef, G. A., De Dreu, C. K., \& Manstead, A. S. (2010). An interpersonal approach to emotion in social decision making: The emotions as social information model. Advances in Experimental Social Psychology, 42, 45-96.

Van Laer, T., De Ruyter, K., \& Cox, D. (2013). A walk-in customers' shoes: How attentional bias modification affects ownership of integrity-violating social media posts. Journal of Interactive Marketing, 27(1), 14-27.

Wang, L., Malhotra, D., \& Murnighan, J. K. (2011). Economics education and greed. Academy of Management Learning \& Education, 10(4), 643-660.

Wang, X., \& Krumhuber, E. G. (2017). The love of money results in objectification. British Journal of Social Psychology, 56(2), 354-372.

Wang, X., \& Krumhuber, E. G. (2018). Mind perception of robots varies with their economic versus social function. Frontiers in Psychology, 9, 1230.

West, S. G., \& Brown, T. J. (1975). Physical attractiveness, the severity of the emergency and helping: A field experiment and interpersonal simulation. Journal of Experimental Social Psychology, 11(6), 531-538.

Wilson, D. W. (1978). Helping behavior and physical attractiveness. The Journal of Social Psychology, 104(2), 313-314.

Wilson, J. P., \& Rule, N. O. (2015). Facial trustworthiness predicts extreme criminalsentencing outcomes. Psychological Science, 26(8), 1325-1331.

Wubben, M. J., De Cremer, D., \& Van Dijk, E. (2009). How emotion communication guides reciprocity: Establishing cooperation through disappointment and anger. Journal of Experimental Social Psychology, 45(4), 987-990. 
Running head: MONEY AND EMOTIONS

Zaleskiewicz, T., Gasiorowska, A., \& Vohs, K. D. (2017). The Psychological Meaning of Money. Economic Psychology, 105-122. 


\section{Footnotes}

1. The following comprehension checks were obtained in a fixed order: "1. Out of your private initial 100 money/candy points, you can put into the joint account. Select the option that is incorrect. Answer options: 0, 20, 50, 80, 100, 120"; "2. If you choose to put 80 money/candy points into the joint account and the other player chooses to put 40 money/candy points into the joint account, how many points can you get for that round? Answer options: 100, 110, 150, 180"; " 3 . If you choose to put 40 money/candy points and the other player chooses to put 80 money/candy points into the joint account, how many points can you get for that round? Answer options: 100, 110, 150, 180"; "4. If you choose to put 0 and the other player chooses to put 100 money/candy points into the joint account. How many points can you get for that round? Answer options: 100, 110, 150, 175"; "5. Regardless of what the other person does, the more you want the other player to earn__ Answer options: The more you put into this joint account. The less you put into this joint account."; “6. Regardless of what the other person does, the more you want yourself to earn_.. Answer options: The more you put into this joint account. The less you put into this joint account." For all comprehension checks, participants received feedback about whether their answers were correct or incorrect. If the question was answered incorrectly, the correct answer was displayed on the screen. 86\% (Study 1), 82\% (Study 2) and 89\% (Study 3) of participants' initial answers were correct. Excluding participants whose initial answers were incorrect (before informing them of the correct ones) did not significantly change the main results.

2. Contribution/Allocation rates in response to targets with a neutral emotion expression were as follows: $M_{\text {money }}=18.7 \%, S D_{\text {money }}=28.4, M_{\text {candy }}=27.5 \%, S D_{\text {candy }}=33.1$ $($ Study 1$) ; M_{\text {money }}=26.3 \%, S D_{\text {money }}=19.7, M_{\text {candy }}=30.4 \%, S D_{\text {candy }}=19.8($ Study 2$) ; M_{\text {money }}$ $=19.5 \%, S D_{\text {money }}=27.5, M_{\text {candy }}=34.5 \%, S D_{\text {candy }}=30.2($ Study 3$) ; M_{\text {money }}=8.77 \%, S D_{\text {money }}$ 
$=14.9, M_{\text {candy }}=19.1 \%, S D_{\text {candy }}=20.0($ Study 4$)$. A meta-analysis showed a main effect of condition (see the meta-analysis section for more details).

3. The following comprehension checks were obtained in a fixed order: " 1 . What is your role in this game? Answer options: Allocator, Receiver"; "2. Which of the following options is incorrect? I can allocate_ money/candy points to a receiver. Answer options: 0, 50, 100, 120"; "3. The more money/candy you keep for yourself in the game_. Answer options: The fewer money/candy points you are going to earn in the end, and thus the less likely you are going to get the money bonus/candy gift certificate. The more money/candy points you are going to earn in the end, and thus the more likely you are going to get the money bonus/candy gift certificate.”; “4 . The more money/candy you keep for yourself in the game_. Answer options: The fewer money/candy your receiver is going to earn in the end. The more money/candy your receiver is going to earn in the end." For all comprehension checks, participants were informed whether their answers were correct or incorrect. If the question was answered incorrectly, the correct answer was provided including detailed explanations. 93\% (Study 4) and 96\% (Study 5) of participants' initial answers were correct. Excluding participants whose initial answers were incorrect (before informing them of the correct answers) did not significantly change the main results.

4. The order in which the questions were posed (before or after allocation) had no effect on participants' impression ratings, $t(715)=-0.80, p=.423, d=-0.05$.

5. The order in which the questions were posed (before or after the game) had no effect on participants' reported levels of self-gain focus, $t(715)=0.06, p=.955, d=0.004$. 


\section{Figure Captions}

Figure 1. Contribution rates by emotion as a function of game condition (Wall Street/money vs. Community/candy) in Study 1. Error bars represent +/- 1SE.

Figure 2. Contribution rates by emotion as a function of game condition (Wall Street/money vs Community/candy) in Study 2. Error bars represent +/- 1SE.

Figure 3. Contribution rates by emotion as a function of game condition (money vs. candy) in Study 3. Error bars represent +/- 1SE.

Figure 4. Allocation levels by emotion as a function of game condition (money vs. candy) in Study 4. Error bars represent +/- 1SE.

Figure 5. Allocation levels by emotion as a function of game condition (money vs. candy) in Study 5. Error bars represent +/- 1SE.

Figure 6. Ratings of self-gain focus by emotion as a function of game condition (money vs. candy) in Study 5. Error bars represent +/- 1SE.

Figure 7. Mediation model for the effect of emotion on allocation via trait impression in Study 5. Values for the indirect path (i.e., when controlling for the mediator) are shown in parenthesis. Sequential coding, anger: D1 = 0, D2 = 0; sadness: D1 = 0, D2 = 1; happiness: $\mathrm{D} 1=1, \mathrm{D} 2=1$

Figure 8. Mediation model for the interaction effect of emotion and condition on allocation via self-gain focus in Study 5. Values for the indirect path (i.e., when controlling for the mediator) are shown in parenthesis. Sequential coding, anger: D1 = 0, D2 =0; sadness: D1 = 0, D2 = 1; happiness: D1 = 1, D2 = 1 
Running head: MONEY AND EMOTIONS

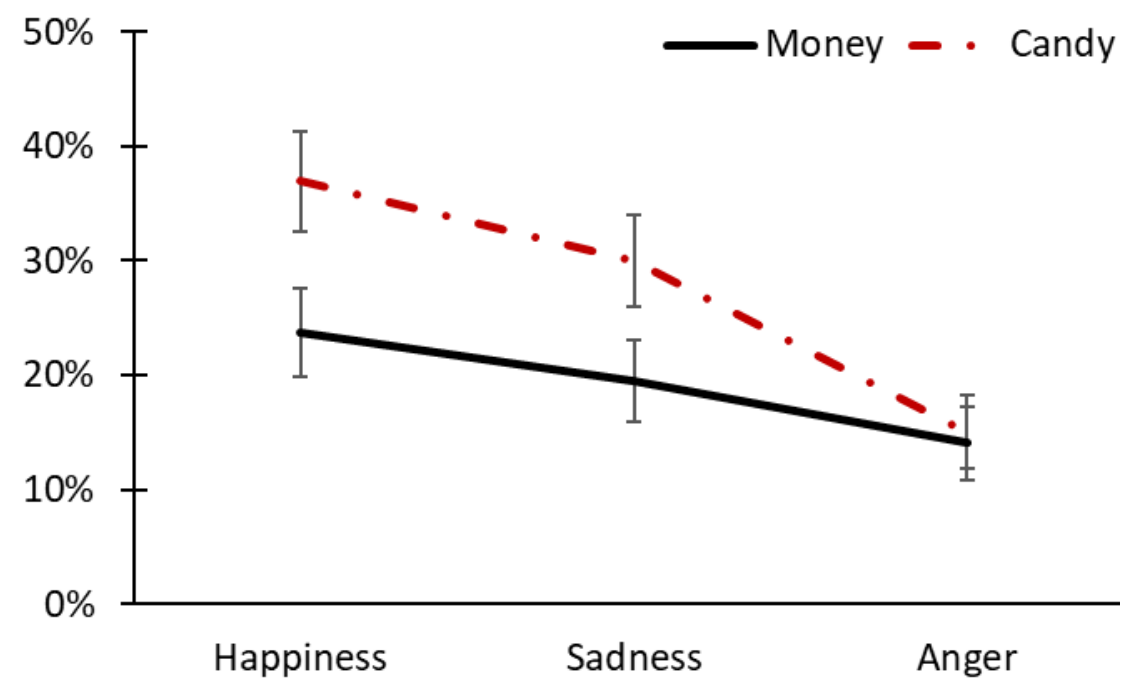


Running head: MONEY AND EMOTIONS

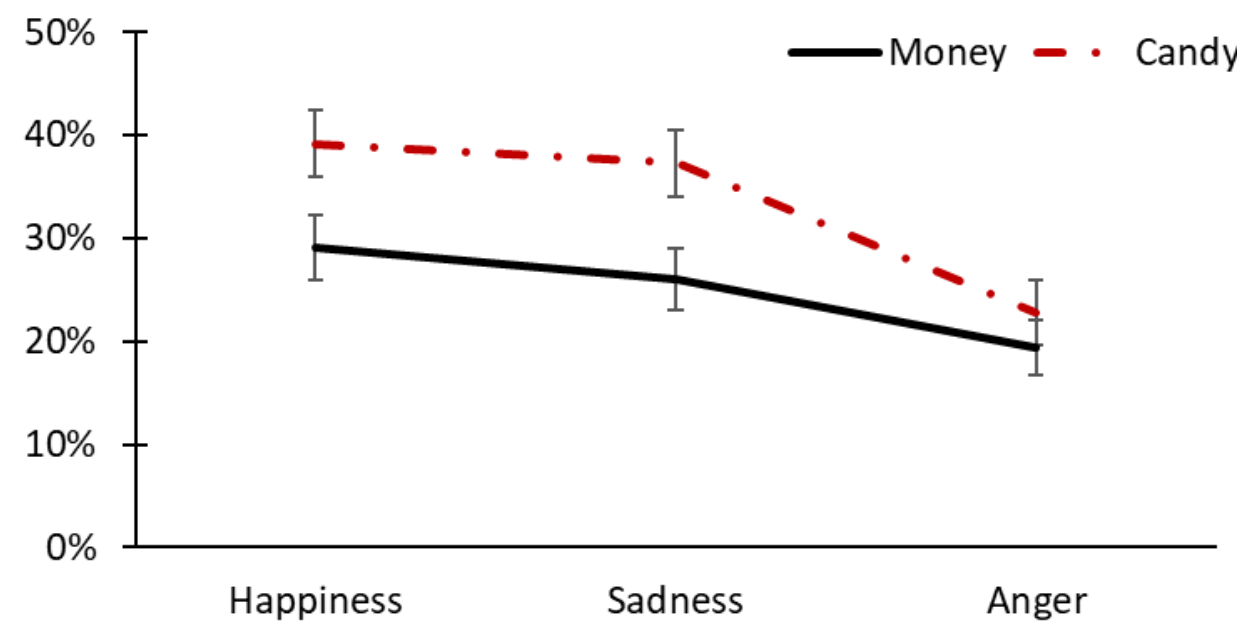


Running head: MONEY AND EMOTIONS

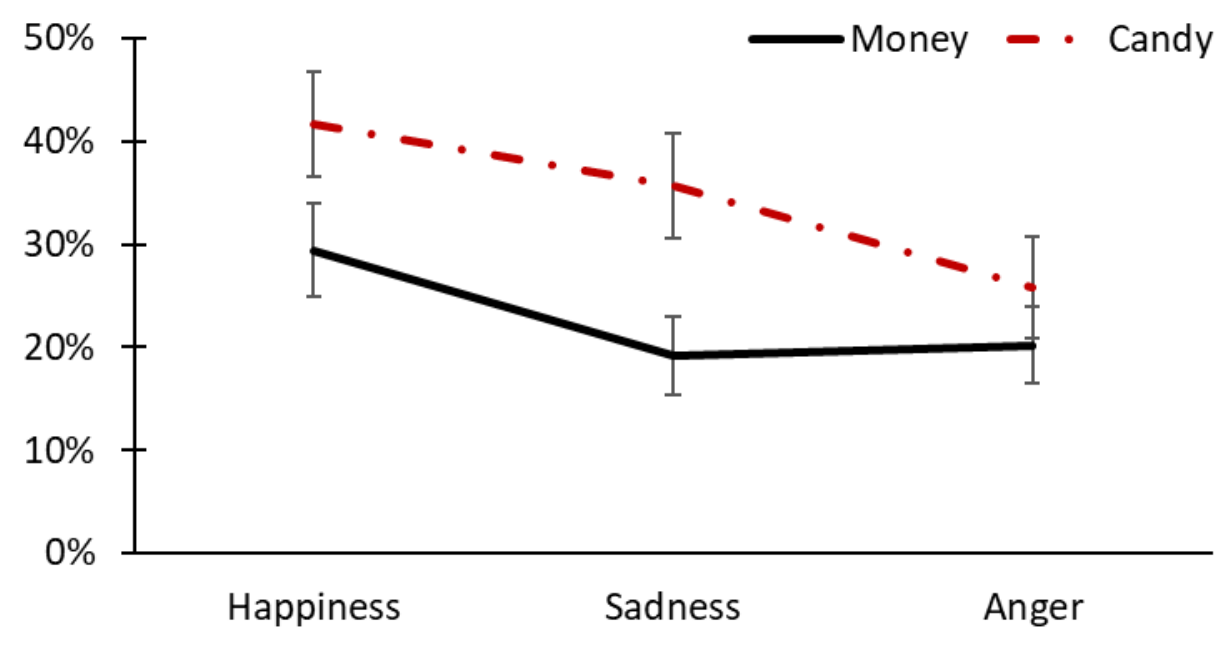


Running head: MONEY AND EMOTIONS

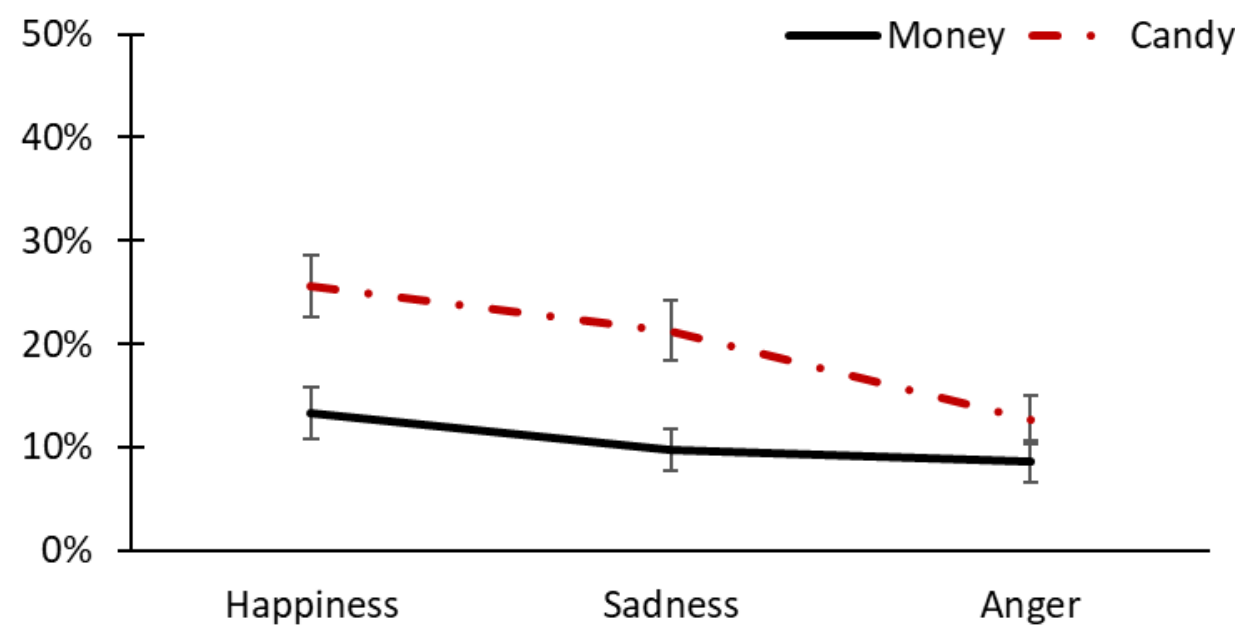


Running head: MONEY AND EMOTIONS

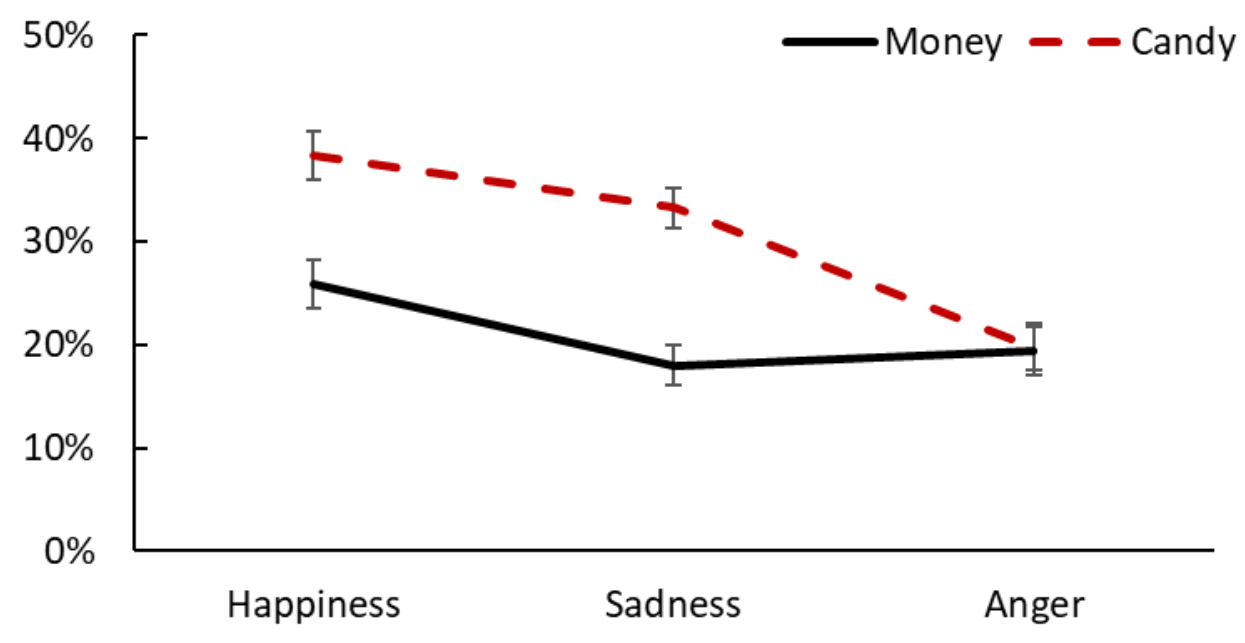


Running head: MONEY AND EMOTIONS

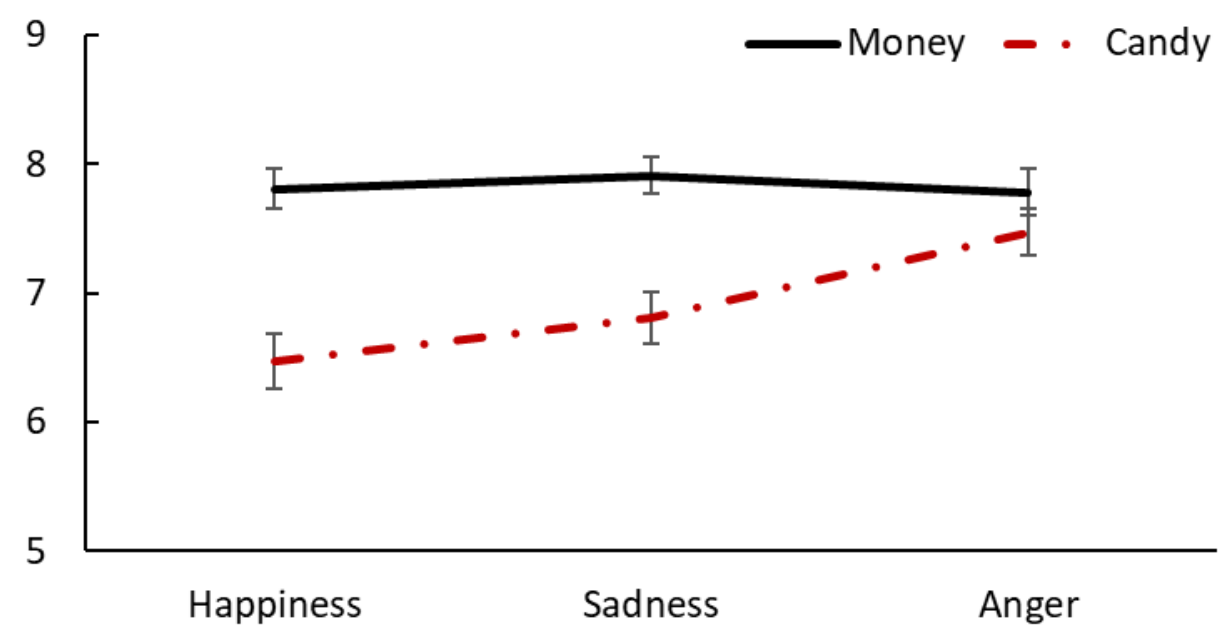


Running head: MONEY AND EMOTIONS

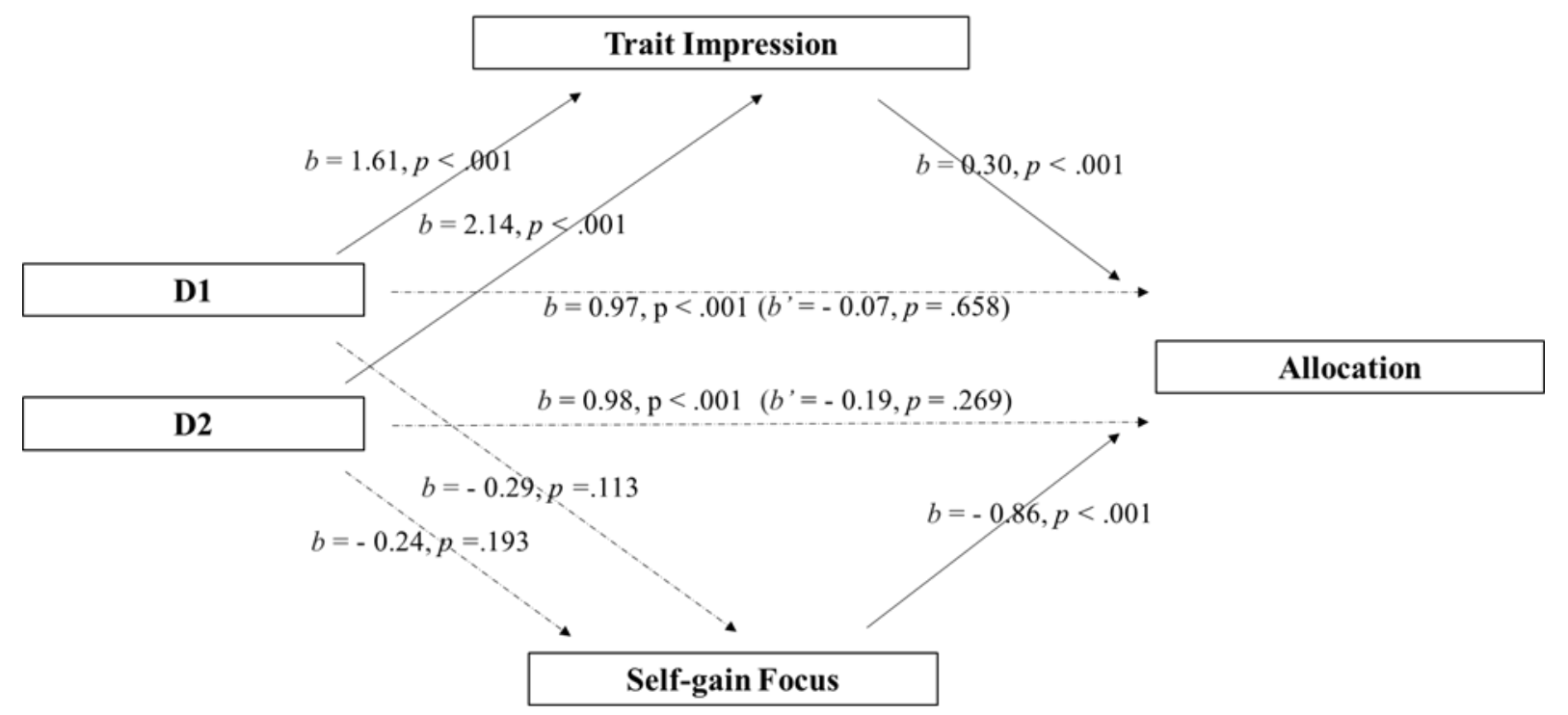




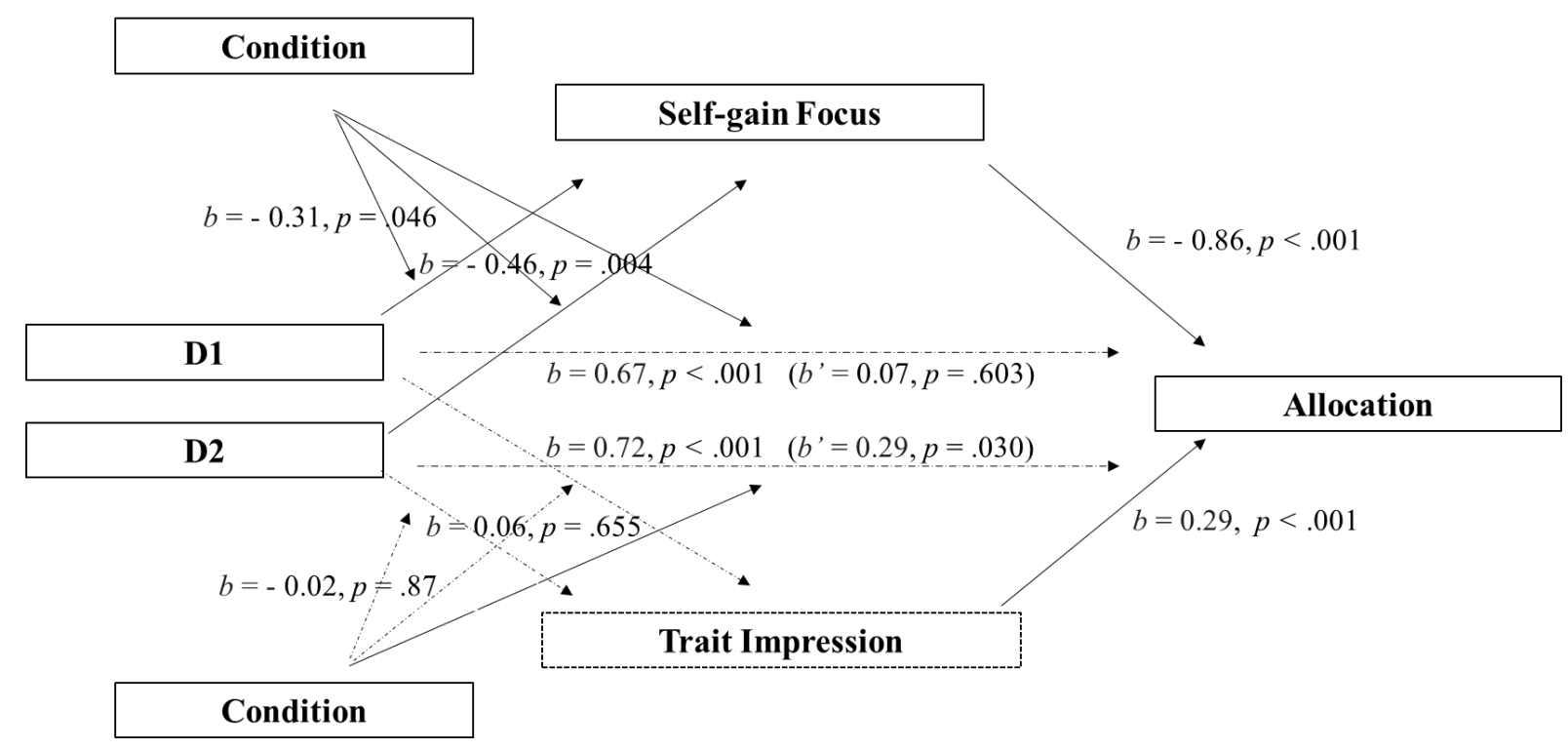

